\title{
Self-Contained Automated Methodology for Optimal Flow Control
}

\author{
Ronald D. Joslin $\Gamma^{1}$ Max D. GunzburgerI ${ }^{2}$ Roy A. Nicolaides ${ }^{\circledR}$ \\ Gordon ErlebacherI $I^{4}$ and M. Yousuff Hussaini ${ }^{5}$
}

\begin{abstract}
This paper describes a self-containedTautomated methodology for active flow control which couples the time-dependent Navier-Stokes system with an adjoint Navier-Stokes system and optimality conditions from which optimal states $\Gamma$ i.e. Tunsteady flow fields and controls (e.g. Tactuators) $\Gamma$ may be determined. The problem of boundary layer instability suppression through wave cancellation is used as the initial validation case to test the methodology. Here the objective of control is to match the stress vector along a portion of the boundary to a given vector; instability suppression is achieved by choosing the given vector to be that of a steady base flow. Control is effected through the injection or suction of fluid through a single orifice on the boundary. The results demonstrate that instability suppression can be achieved without any a priori knowledge of the disturbance which is significant because other control techniques have required some knowledge of the flow unsteadiness such as frequencies $\Gamma$ instability type $\Gamma$ etc. The present methodology has been extended to three dimensions and may potentially be applied to separation control $\Gamma$ re-laminarization $\Gamma$ and turbulence control applications using one to many sensors and actuators.
\end{abstract}

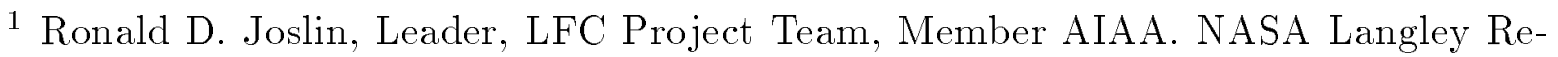
search CenterTMail Stop 170ГHamptonГVA 23681-0001.

${ }^{2}$ Consultant $\Gamma$ Institute for Computer Applications in Science and Engineering; cur-

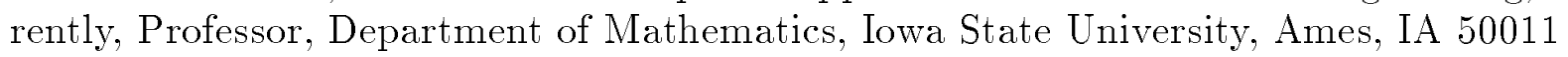

${ }^{3}$ Consultant $\Gamma$ Institute for Computer Applications in Science and Engineering; currently $\Gamma$ Professor $\Gamma$ Department of Mathematics $\Gamma$ Carnegie Mellon University $\Gamma$ Pittsburgh $\Gamma$ PA 15213

4 Assistant Professor of Mathematics $\Gamma$ Member AIAA. Florida State UniversityГTallahasseeTFL 32306-4052

${ }^{5}$ Eminent Scholar Chair in High Performance Computing TFellow AIA A. Florida State University TTallahasseeГFL $32306-4052$ 


\section{Introduction}

In the last decadeTincreased attention has been devoted to the development of techniques capable of enhancing our ability to control the unsteady flow in a wide variety of configurations such as engine inlets and nozzles $\Gamma$ combustors $\Gamma$ automobiles $\Gamma$ aircraft $\Gamma$ and marine vehicles. Controlling the flow in these configurations can lead to greatly improved efficiency and performance $\Gamma$ while decreasing the noise levels generally associated with the otherwise unattended unsteady flow. Depending on the desired result Tone might wish to delay or accelerate transition Treduce drag or enhance mixing. There might be a need to postpone flow-separation Tincrease lift or manipulate a turbulence field. Gad-el-Hak ${ }^{1}$ and Gad-el-Hak and Bushnel1 ${ }^{2}$ provide an excellent introduction to and overview of various control methodologies.

Small improvements in system performance often lead to large payoffs. For example Butter $^{3}$ estimates that a 5 percent improvement in landing maximum lift coefficient $\left(C_{l}(\max )\right)$ can translate to a 25 percent increase in payload. Cousteix ${ }^{4}$ notes that 45 percent of the drag for a commercial transport transonic aircraft is due to skin friction on the wings $\Gamma$ fuselage $\Gamma$ fin $\Gamma$ etc. $\Gamma$ and that a 10 to 15 percent reduction of the total drag can be expected by laminarizing the flow over the wings and the fin. This translates into a reduction in fuel requirements $\Gamma$ improved performance $\Gamma$ and/or increased payload. Muirhead ${ }^{5}$ has shown with a wind tunnel investigation that control of flow separation on a tractortrailor truck can reduce the drag by 30 to 40 percent of the baseline truck configuration. This translates into a savings of millions of barrels of fuel per year.

Encouraged by the potential for huge rewards with what may be a modest input $\Gamma$ research into ways of achieving the above gains is ever important. In many applications $\Gamma$ the flow starts from a smooth laminar state $\Gamma$ which is inherently unstable $\Gamma$ and develops instability waves. These instability waves grow exponentially nonlinearly interacting $\Gamma a n d$ lead ultimately to fully developed turbulence or flow seperation. Therefore $\Gamma$ one goal of a good control system is to inhibit $\Gamma$ if not eliminate $\Gamma$ these instabilities which lead to the deviation from laminar to turbulent flow state.

\subsection{The Wave-Cancellation Concept}

The simplest form of control which might achieve this objective is the wavecancellation approach $\Gamma$ based on the premise that the instability mechanisms in low-speed transition are dominated by a single instability wave; therefore $\Gamma$ cancelling this wave will preclude the nonlinear interactions leading to laminar-turbulent transition. The wavecancellation method further assumes that a wavelike disturbance can be cancelled by in- 
troducing another wave equal in amplitude but opposite in phase $\Gamma$ and thus it is mostly applicable to systems governed by linear or quasi-linear equations. The key is to determine the parameters of the downstream wave which counter (cancel) the evolution of the upstream generated wave. Because there are a number of experiments and numerical simulations which validate this approach $\Gamma$ the wave-cancellation problem is an excellent test problem for the new flow-control methodology described in this paper.

Most of the experiments $\Gamma$ aimed at verifying the wave-cancellation concept $\Gamma$ were conducted on the flat plate except those of Ladd and Hendricks ${ }^{6} \Gamma$ Pupator and Saric ${ }^{7} \Gamma$ and $\operatorname{LaddI}^{8}$ who considered axisymmetric bodies. Many of these experiments were conducted in water tunnels. Vibrating wires $\mathbb{P}^{3}$ hot strips $\Gamma^{10,11}$ suction and blowing $\bar{\Gamma}^{\overline{8}}$ electromagnetic generators $\mathrm{I}^{2}$ and adaptive heating ${ }^{6}$ are some of the methods that were used to generate the controlling wave. All these input mechanisms give the necessary control of the phase and amplitude of the input wave. Among the more successful studies $\Gamma$ Milling ${ }^{9}$ and Thomas $^{12}$ achieved at least an 80 percent reduction in the input amplitude of the 2-D wave (with 0.6\%-1\% amplitude). However $\Gamma$ it was not possible to achieve relaminarization $\Gamma$ probably because of the three-dimensionality of the flow resulting from the interaction between background disturbances and the primary 2-D wave. As expected $\Gamma$ the studies conducted on axisymmetric bodies produced relatively less wave cancellation because these flows are inherently three-dimensional. Furthermore $\operatorname{good}$ wave cancellation requires a linear system with constant coefficients. This requirement is clearly violated for flow over a body

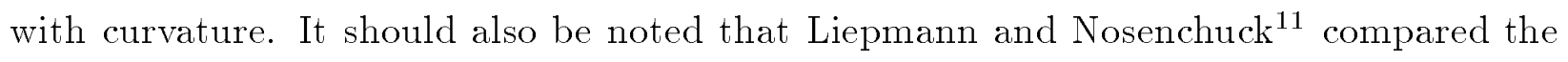
effects of steady and non-steady heating $\Gamma$ and found that steady heating demands a $2000 \%$ increase in energy over an unsteady wave-cancellation technique. Hence $\Gamma$ unsteady control may be more efficient for flow control applications.

In addition to the aforementioned experiments $\Gamma$ several theoretical (i.e. $\Gamma$ linear computations and theory) and computational studies (i.e Tnonlinear simulations) have focused on understanding the physics of this wave-cancellation process. Maestrello and Ting ${ }^{13}$ provided a linear asymptotic analysis to demonstrate the relationship between the input of localized disturbances and their effect on the Tollmien-Schlichting (TS) instability waves present in the wall-bounded shear flow. They showed that small amounts of local periodic heating could excite disturbances that actively control the TS waves which propagate in a boundary layer on a flat plate. Analogous to the experiments $\Gamma$ several wave input mechanisms were considered. In one of the early Navier-Stokes simulations of active control $\Gamma$ Biringen ${ }^{14}$ used suction and blowing at the wall in a channel flow. He observed approximately a 50 percent reduction in the amplitudes of the 2-D instabilities and a decrease 
in the growth of the 3-D instabilities. The Reynolds stress originally generated by the waves was all but removed. On the other handГMetcalfe et al. ${ }^{15}$ studied the effect of a moving wall on unstable waves traveling in a laminar flow on a flat plate. The simulations were based on the Navier-Stokes equations solved within a temporal framework. An energy analysis revealed that the wall motion causes the Reynolds-stress term to become negative $\Gamma$ which implies a feed of energy from the perturbed flow back into the mean flow. In effect $\Gamma$ this energy analysis showed how a perturbation to an unstable flow can be stabilizing. HoweverTan instability wave eventually formed downstream of the control $\Gamma$ with the same growth rate as the uncontrolled wave. This is a clear indication that the cancellation was not complete. Although intuitively obvious $\Gamma$ until the work of Bower et al. ${ }^{16}$ and Pal et al. $\Gamma^{17}$ it was not known that perfect cancellation could be obtained within the context of linear theory (for which the mean flow is independent of the propagating direction). They used the 2-D Orr-Sommerfeld equation to study and control instability-wave growth by superposition Tand showed $\Gamma$ within the limits of linear stability theory and the parallel-flow assumption $\Gamma$ that single and multifrequency waves can be cancelled. The basic conclusions drawn by the early experimentalists were confirmed by the studies of Laurien and Kleiser ${ }^{18}$ and Kral and Fasel. ${ }^{19}$ They showed that it was possible to delay/accelerate transition by superposing disturbances out of/in phase with the primary TS wave. Similar results were also reported by Danabasoglu et al. ${ }^{20}$ Finally $\mathrm{J}$ Joslin et al. ${ }^{21}$ performed a numerical experiment which served to unequivocally demonstrate the link between linear superposition and instability suppression. To ensure that linear superposition of individual instabilities was $\Gamma$ in fact $\Gamma$ responsible for the results found in previous experiments and computations $\Gamma$ they carried out three simulations with i) only the disturbance; ii) only the control; and iii) using both disturbance and control which is the wave-cancellation case. By discretely summing the control-only and forcing-only numerical results $\Gamma$ they found that this linear superposed solution is identical to the wave-cancellation results. These tests cleary verify the hypothesis that linear superposition is the reason for the previous experimental and computational results.

¿From the above experimentsTlinear computations and nonlinear simulations $\Gamma$ several common features emerge: (1) It is impossible to achieve perfect wave cancellation unless the system is linear $\Gamma$ with constant coefficients; (2) the efficiency of wave cancellation decreases as the system becomes more nonlinear; (3) as the geometry of the configuration becomes more complex Tcancellation becomes more difficult; and (4) the current approaches require foreknowledge of the instability wave characteristics $\Gamma$ such as its frequency and amplitude before wave suppression can be realized. 


\subsection{Optimal Control Theory}

The optimal control theory provides an approach which does not require a priori knowledge of the flow characteristics. The goal of optimal control theory is to minimize or maximize an objective function in a robust manner. When the flow is time-dependent Tand a strong function of initial conditions $\Gamma i t$ becomes difficult to establish the precise controls that will achieve the desired effect. Wave-cancellation $\Gamma$ as discussed above $\Gamma$ only works well when the input wave has a dominant frequency and its properties are known. Then (either in a passive fashion $\Gamma$ or through a feedback mechanism) $\Gamma$ one seeks to cancel its effect while still in a linear regime. In practice $\Gamma$ there are many waves $\Gamma$ which can interact nonlinearly in ways not always known in advance. Rather than try to cancel the incoming wavesTone seeks appropriate controls in other ways. One means of achieving this Twithout an extensive search over the space of possible controls $\Gamma$ is to postulate a family of desired controls (e.g. Tan arbitrary time-dependent amplitude and a specified spatial distribution) $\Gamma$ and an objective function (i.e. Tstress over a region of the plate). Then $\Gamma$ through a formal minimization process $\Gamma$ one derives a set of differential equations $\Gamma$ and their adjoints $\Gamma$ whose solution produces the optimal actuator profile (among the specified set). While the solution to this set of equations cannot be accomplished in real time $\Gamma$ the results can be applied using standard passive or active control mechanisms. The advantage of this approach is that entire collections of controls can be studied simultaneously Trather than one at a time.

Optimal control methodologies have been recently applied to a variety of problems involving drag reduction Tflow and temperature matching Tetc. to provide more sophisticated flow control strategies in engineering applications. Computational fluid dynamics (CFD) algorithms have reached a sufficiently high level of maturity $\Gamma$ generality $\Gamma$ and efficiency so that it is now feasible to implement sophisticated flow optimization methods $\Gamma$ which lead to a large number of coupled partial differential equations. Optimal control theory is quite mathematical $\Gamma$ and its formal nature is amenable to the derivation of mathematical theorems related to existence of solutions and well-posedness of the problem. (Only partial results of this type are possible in three-dimensions since $\Gamma$ in this case $\Gamma$ the Navier-Stokes equations themselves do not enjoy a full theoretical foundation; in two-dimensions $\Gamma$ a complete theory is available.) Two recent surveys of the mathematical theories of optimal flow control are Gunzburger ${ }^{22}$ and Borggaard et al. ${ }^{23}$ A mathematical study of a simplified problem related to the one considered in this paper can be found in Fursikov et al. ${ }^{24}$

Flows may also be controlled through controllers whose actuation is determined by feedback laws. For unsteady flows $\Gamma$ including turbulent flows $\Gamma$ feedback controls are considered $\Gamma$ for example $\Gamma$ in Choi et $a 1 .{ }^{25}$ and Bewley et al. ${ }^{26} \Gamma$ as well as in Joslin et al. ${ }^{28}$ 
Determining optimal feedback laws is a very difficult propositionTespecially in the context of nonlinear problems so that one usually has to be content with using sub-optimal feedback laws. On the other hand in our study $\Gamma$ we consider optimal controllers as determined by optimal control methodologies instead of a feedback strategy.

Optimal control techniques will not provide the real time control that one is ultimately interested in Tbut by systematically computing the best control within specified tolerances $\Gamma$ with a given objective function $\Gamma i t$ will be possible to develop strategies (active or passive) to control a wide variety of disturbances. For exampleTto effectively control boundary-layer transition due to the interaction of a crossflow vortex and a TS wave using periodic heating and cooling Toptimal control would allow: (1) a determination of the best objective function to use for a given type of control (some are better than others) and (2) provide insight into the relationship between the time dependence of the control and the input waves. This insight could then be built into a neural network Tor other type of self-learning system $\Gamma$ to allow effective control over a wide range of input parameters.

\subsection{The Current Approach}

The methodology of the current paper is based on defining a control mechanism and an objective for control $\Gamma$ and then finding $\Gamma i n$ a systematic and automated mannerTcontrols that best meet the objective. In the present setting $\Gamma$ an objective or cost functional is defined that measures the "distance" between the measured stresses $\Gamma$ and their desired values along a limited section of the bounding wall and over a specified length of time. One may interpret the objective functional as a "sensorT" i.e. The objective functional senses how far the flow stresses along the wall are from the corresponding desired values. To control the flow Ttime-dependent injection and suction are imposed along a small orifice in the bounding wall. Although the spatial dependence of the suction profile is specified (for simplicity) The optimal control methodology determines the time-variation of this profile. However $\Gamma$ unlike feedback control methodologies wherein the sensed data determines the control through a specified feedback law or controller $\Gamma$ here the time-dependence of the control is the natural result of the minimization of the objective functional. This scenario is shown in Fig. 1. We have a sensor that feeds information to a controller that in turn feeds information to the actuator. However $\Gamma$ in the optimal control setting $\Gamma$ the sensor is actually an objective functional and the controller is a coupled system of partial differential equations that determine the control that does the best job of minimizing the objective functional. The present active-control approach is demonstrated for the evolution and

automated control of spatially growing $2 \mathrm{D}$ disturbances in a flat-plate boundary layer. As the length of time over which the minimization process is increased $\Gamma$ the results obtained by 
wave cancellation are recovered $\Gamma$ thus validating the approach. The ultimate goal of this line of research is to introduce automated control to external flows over realistic configurations such as wings and fuselages $\Gamma$ and to devise novel flow control systems.

\section{The Optimization Problem}

\subsection{The state equations}

Let $\Omega$ denote the flow domain which is the semi-infinite channel or boundary layer $[x \geq 0 \Gamma 0 \leq y \leq h] \Gamma$ where $h$ is the location of the upper wall for the channel or the truncated freestream distance for the boundary layer. Let $\Gamma$ denote its boundary and let $(0, T)$ be the time interval of interest. The inflow part of the boundary $[x=0,0 \leq y \leq h]$ is denoted by $\Gamma_{i}$ and the part of the boundary on which control is applied (i.e. $\Gamma$ along which the suction and blowing actuator is placed) by $\Gamma_{a}$ which is assumed to be a finite connected part of the lower boundary (or wall) $[x \geq 0 \Gamma y=0]$. Solid walls are denoted by $\Gamma_{w}$; for the channel flow $\Gamma \Gamma_{w}$ is the lower boundary $[x \geq 0 \Gamma y=0]$ with $\Gamma_{a}$ excluded and the upper boundary $[x \geq 0 \Gamma y=h]$; for the boundary layer flow $\Gamma \Gamma_{w}$ is only the lower boundary with $\Gamma_{a}$ excluded. For the boundary-layer case $\Gamma$ the upper boundary $[x \geq 0 \Gamma$ $y=h] \Gamma$ which is not part of $\Gamma_{w} \Gamma$ is denoted by $\Gamma_{e}$. Controls are only activated over the given time interval $t \in\left(T_{0}, T_{1}\right) \Gamma$ where $0 \leq T_{0}<T_{1} \leq T$.

The flow field is described by the velocity vector $(u, v)$ and the scalar pressure $p$ and is obtained by solving the following momentum and mass conservation equations

$$
\begin{gathered}
\frac{\partial u}{\partial t}+u \frac{\partial u}{\partial x}+v \frac{\partial u}{\partial y}+\frac{\partial p}{\partial x}-\nu \frac{\partial}{\partial x}\left(2 \frac{\partial u}{\partial x}\right)-\nu \frac{\partial}{\partial y}\left(\frac{\partial u}{\partial y}+\frac{\partial v}{\partial x}\right)=0 \text { in }(0, T) \times \Omega, \\
\frac{\partial v}{\partial t}+u \frac{\partial v}{\partial x}+v \frac{\partial v}{\partial y}+\frac{\partial p}{\partial y}-\nu \frac{\partial}{\partial x}\left(\frac{\partial u}{\partial y}+\frac{\partial v}{\partial x}\right)-\nu \frac{\partial}{\partial y}\left(2 \frac{\partial v}{\partial y}\right)=0 \quad \text { in }(0, T) \times \Omega, \\
\frac{\partial u}{\partial x}+\frac{\partial v}{\partial y}=0 \quad \text { in }(0, T) \times \Omega,
\end{gathered}
$$

subject to initial and boundary conditions:

$$
\begin{gathered}
\left.(u, v)\right|_{t=0}=\left(u_{0}, v_{0}\right) \text { in } \Omega \\
\left.(u, v)\right|_{\Gamma_{a}}=\left\{\begin{array}{l}
\left(g_{1}, g_{2}\right) \text { in }\left(T_{0}, T_{1}\right) \\
(0,0) \text { in }\left(0, T_{0}\right) \text { and }\left(T_{1}, T\right)
\end{array}\right. \\
\left.(u, v)\right|_{\Gamma_{i}}=\left(u_{i}, v_{i}\right) \text { and }\left.(u, v)\right|_{\Gamma_{w}}=(0,0) \text { in }(0, T) \\
(u, v, p) \rightarrow \text { base flow, } \frac{\partial u}{\partial x}, \frac{\partial v}{\partial x} \rightarrow 0 \quad \text { as } x \rightarrow \infty .
\end{gathered}
$$


Here $\Gamma$ the initial velocity vector $\left(u_{0}(x, y), v_{0}(x, y)\right)$ and the inflow velocity vector $\left(u_{i}(t, y), v_{i}(t, y)\right)$ are assumed given and the base flow is assumed to be Poiseuille flow for the channel case and Blasius flow for the boundary-layer case. The above system holds for both the channel and Blasius flow cases; in the latter case $\Gamma$ the upper boundary is not part of $\Gamma_{w}$ and the additional boundary condition

$$
\left.u\right|_{\Gamma_{e}}=U_{\infty} \quad \text { and } \quad p-\left.2 \nu \frac{\partial v}{\partial y}\right|_{\Gamma_{e}}=P_{\infty} \quad \text { in } \quad(0, T)
$$

is imposed $\Gamma$ where $U_{\infty}$ and $P_{\infty}$ denote the free-stream flow speed and pressure $\Gamma$ respectively.

The control functions $g_{1}(t, x)$ and $g_{2}(t, x)$ which give the rate at which fluid is injected or sucked tangentially and perpendicularly $\Gamma$ respectively $\Gamma$ through $\Gamma_{a}$ are to be determined as part of the optimization process. In order to make sure that the control remains bounded at $T_{0} \Gamma$ it is required that

$$
\left.g_{1}\right|_{t=T_{0}}=g_{10}(x) \text { and }\left.g_{2}\right|_{t=T_{0}}=g_{20}(x) \quad \text { on } \Gamma_{a} \text {, }
$$

where $g_{10}(x)$ and $g_{20}(x)$ are specified functions defined on $\Gamma_{a}$. Commonly $\Gamma$ one chooses $g_{10}(x)=g_{20}(x)=0$.

\subsection{The objective functional and the optimization problem}

Assume that $\Gamma_{s}$ is a finite $\Gamma$ connected part of the lower boundary $[x \geq 0 \Gamma y=0]$ which is disjoint from $\Gamma_{a}$ and that $\left(T_{a}, T_{b}\right)$ is a time interval such that $0 \leq T_{a}<T_{b} \leq T$. Then $\Gamma$ consider the functional

$$
\begin{gathered}
\mathcal{J}\left(u, v, p, g_{1}, g_{2}\right)=\frac{\alpha_{1}}{2} \int_{T_{a}}^{T_{b}} \int_{\Gamma_{s}}\left|\tau_{1}-\tau_{a}\right|^{2} d \Gamma d t+\frac{\alpha_{2}}{2} \int_{T_{a}}^{T_{b}} \int_{\Gamma_{s}}\left|\tau_{2}-\tau_{b}\right|^{2} d \Gamma d t \\
+\frac{\beta_{1}}{2} \int_{T_{0}}^{T_{1}} \int_{\Gamma_{a}}\left(\left|\frac{\partial g_{1}}{\partial t}\right|^{2}+\left|g_{1}\right|^{2}\right) d \Gamma d t+\frac{\beta_{2}}{2} \int_{T_{0}}^{T_{1}} \int_{\Gamma_{a}}\left(\left|\frac{\partial g_{2}}{\partial t}\right|^{2}+\left|g_{2}\right|^{2}\right) d \Gamma d t
\end{gathered}
$$

where $g_{1}$ and $g_{2}$ denote the controls and $\tau_{a}(t, x)$ and $\tau_{b}(t, x)$ are given functions defined on $\left(T_{a}, T_{b}\right) \times \Gamma_{s}$. Note that since $\Gamma_{s}$ is part of the lower boundary of the channel or boundary-layer wall $\tau_{1}=\nu \partial u / \partial y$ and $\tau_{2}=-p+2 \nu \partial v / \partial y$ are the shear and normal stresses $\Gamma$ respectively $\Gamma$ exerted by the fluid on the bounding wall along $\Gamma_{s}$ and thus $\tau_{a}$ and $\tau_{b}$ may be interpreted as given shear and normal stresses $T$ repectively. Then $\Gamma$ the boundary segment $\Gamma_{s}$ can be thought of as a sensor which measures the stresses on the wall. Thus $\Gamma$ in $(10) \Gamma \Gamma_{s}$ is the part of the boundary $\Gamma$ along which one wishes to match the shear and normal stresses to the given functions $\tau_{a}$ and $\tau_{b} \Gamma$ respectively $\Gamma$ and $\left(T_{a}, T_{b}\right)$ is the time 
interval over which this matching is to take place. (There are no diffilcultiesTother than notational introduced if one wishes to match each component of the stress vector over a different boundary segment and/or over a different time interval.)

The third and fourth terms in (10) are used to limit the size of the control. Indeed bounds are a priori placed on $g_{1}$ or $g_{2}$; their magnitudes are limited by adding a penalty to the stress matching functional defined by the first two terms in (10). The particular form that these penalty terms take $\mathrm{i}$ i.e $\Gamma$ the third and fourth terms in (10) $\Gamma$ is motivated by the necessity to limit not only the size of the controls $g_{1}$ and $g_{2} \Gamma$ but also to limit oscillations. The constants $\alpha_{1} \Gamma \alpha_{2} \Gamma \beta_{1} \Gamma$ and $\beta_{2}$ can be used to adjust the relative importance of the terms appearing in the functional (10).

The (constrained) optimization problem is given as follows:

Find $u, v, p, g_{1}$, and $g_{2}$ such that the functional $\mathcal{J}\left(u, v, p, g_{1}, g_{2}\right)$ given in (10) is minimized subject to the requirement that (1)-(7) and (10) are satisfied and, for the boundary-layer flow case, (8) is also satified.

\section{The Optimality System}

We first consider Tin Sections 3.1-3.5 Tthe case of a channel flow; the optimality system for the boundary layer flow is considered in Section 3.6.

\subsection{The Lagrangian Functional}

The method of Lagrange multipliers is formally used to enforce the constraints (1)-(3) and (5). To this end $\Gamma$ the Lagrangian functional 


$$
\begin{aligned}
\mathcal{L}\left(u, v, p, g_{1}, g_{2}, \hat{u}, \hat{v}, \hat{p}, s_{1}, s_{2}\right) & \frac{\alpha_{1}}{2} \int_{T_{a}}^{T_{b}} \int_{\Gamma_{s}}\left|\tau_{1}-\tau_{a}\right|^{2} d \Gamma d t+\frac{\alpha_{2}}{2} \int_{T_{a}}^{T_{b}} \int_{\Gamma_{s}}\left|\tau_{2}-\tau_{b}\right|^{2} d \Gamma d t \\
+ & \frac{\beta_{1}}{2} \int_{T_{0}}^{T_{1}} \int_{\Gamma_{a}}\left(\left|\frac{\partial g_{1}}{\partial t}\right|^{2}+\left|g_{1}\right|^{2}\right) d \Gamma d t+\frac{\beta_{2}}{2} \int_{T_{0}}^{T_{1}} \int_{\Gamma_{a}}\left(\left|\frac{\partial g_{2}}{\partial t}\right|^{2}+\left|g_{2}\right|^{2}\right) d \Gamma d t \\
- & \int_{0}^{T} \int_{\Omega} \hat{u}\left(\frac{\partial u}{\partial t}+u \frac{\partial u}{\partial x}+v \frac{\partial u}{\partial y}+\frac{\partial p}{\partial x}-\nu \frac{\partial}{\partial x}\left(2 \frac{\partial u}{\partial x}\right)-\nu \frac{\partial}{\partial y}\left(\frac{\partial u}{\partial y}+\frac{\partial v}{\partial x}\right)\right) d \Omega d t \\
- & \int_{0}^{T} \int_{\Omega} \hat{v}\left(\frac{\partial v}{\partial t}+u \frac{\partial v}{\partial x}+v \frac{\partial v}{\partial y}+\frac{\partial p}{\partial y}-\nu \frac{\partial}{\partial x}\left(\frac{\partial u}{\partial y}+\frac{\partial v}{\partial x}\right)-\nu \frac{\partial}{\partial y}\left(2 \frac{\partial v}{\partial y}\right)\right) d \Omega d t \\
- & \int_{0}^{T} \int_{\Omega} \hat{p}\left(\frac{\partial u}{\partial x}+\frac{\partial v}{\partial y}\right) d \Omega d t \\
- & \int_{T_{0}}^{T_{1}} \int_{\Gamma_{a}} s_{1}\left(u-g_{1}\right) d \Gamma d t-\int_{0}^{T_{0}} \int_{\Gamma_{a}} s_{1} u d \Gamma d t-\int_{T_{1}}^{T} \int_{\Gamma_{a}} s_{1} u d \Gamma d t \\
- & \int_{T_{0}}^{T_{1}} \int_{\Gamma_{a}} s_{2}\left(v-g_{2}\right) d \Gamma d t-\int_{0}^{T_{0}} \int_{\Gamma_{a}} s_{2} v d \Gamma d t-\int_{T_{1}}^{T} \int_{\Gamma_{a}} s_{2} v d \Gamma d t
\end{aligned}
$$

is introduced. In (11) $\Gamma \hat{u}$ and $\hat{v}$ are Lagrange multipliers that are used to enforce the $x$ and $y$-components of the momentum equation (1) and (2) $\Gamma$ respectively $\hat{p}$ is a Lagrange multiplier that is used to enforce the continuity equation (3) $\Gamma$ and $s_{1}$ and $s_{2}$ are Lagrange multipliers that are used to enforce the $x$ and $y$-components of the boundary condition (5) Trespectively. Note that Lagrange multipliers have not been introduced to enforce the constraints $(4) \Gamma(6) \Gamma(7) \Gamma$ and $(9) \Gamma$ so that these conditions must be required of all candidate functions $u \Gamma v \Gamma p \Gamma g_{1} \Gamma$ and $g_{2}$.

Through the introduction of Lagrange multipliers $\Gamma$ the constrained optimization problem is converted into the unconstrained problem:

Find $u, v, p, g_{1}, g_{2}, \hat{u}, \hat{v}, \hat{p}, s_{1}$, and $s_{2}$ satisfying (4), (6), (7), and (9) such that the Lagrangian functional $\mathcal{L}\left(u, v, p, g_{1}, g_{2}, \hat{u}, \hat{v}, \hat{p}, s_{1}, s_{2}\right)$ given by (11) is rendered stationary.

In this problem Teach argument of the Lagrangian functional is considered to be an independent variable so that each may be varied independently.

The first-order necessary condition that stationary points must satisfy is that the first variation of the Lagrangian with respect to each of its arguments vanishes at those points. One easily sees that the vanishing of the first variations with respect to the Lagrange 
multipliers recovers the constraint equations (1)-(3) and (5). Specifically $\Gamma$

$$
\begin{aligned}
& \frac{\delta \mathcal{L}}{\delta \hat{u}}, \frac{\delta \mathcal{L}}{\delta \hat{v}}=0 \quad \Longrightarrow \quad x \text { - and } y \text {-momentum equations (1) and (2) } \\
& \frac{\delta \mathcal{L}}{\delta \hat{p}}=0 \quad \Longrightarrow \quad \text { continuity equation }(3) \\
& \frac{\delta \mathcal{L}}{\delta s_{1}}, \frac{\delta \mathcal{L}}{\delta s_{2}}=0 \quad \Longrightarrow \quad x \text { and } y \text { components of the boundary condition }(5) \text {, }
\end{aligned}
$$

where $\delta \mathcal{L} / \delta \hat{u}$ denotes the first variation of $\mathcal{L}$ with respect to $\hat{u}$ Tetc.

\subsection{The Adjoint Equations}

Next $\Gamma$ set the first variations of the Lagrangian with respect to the state variables $u \Gamma$ $v$ Tand $p$ equal to zero. These result in the adjoint or co-state equations. Note that $\Gamma$ since for the channel flow $\Gamma$ candidate solutions must satisfy $(4) \Gamma(6) \Gamma(7) \Gamma$ and $(9) \Gamma$ one has that

$$
\begin{aligned}
& \left.\delta u\right|_{t=0}=\left.\delta v\right|_{t=0}=0 \text { on } \Omega,\left.\delta g_{2}\right|_{t=T_{0}}=0 \text { on } \Gamma_{a}, \\
& \left.\delta u\right|_{\Gamma_{i}}=\left.\delta v\right|_{\Gamma_{i}}=0 ;\left.\delta u\right|_{\Gamma_{w}}=\left.\delta v\right|_{\Gamma_{w}}=0 \text { for }(0, T), \\
& \delta p, \delta u, \delta v, \frac{\partial \delta u}{\partial x}, \frac{\partial \delta v}{\partial x} \rightarrow 0 \text { as } x \rightarrow \infty \text { for }(0, T)
\end{aligned}
$$

FirstTconsider $\delta \mathcal{L} / \delta p=0$ which yields

$$
\alpha_{2} \int_{T_{a}}^{T_{b}} \int_{\Gamma_{s}} \delta p\left(\tau_{2}-\tau_{b}\right) d \Gamma+\int_{0}^{T} \int_{\Omega}\left(\hat{u} \frac{\partial \delta p}{\partial x}+\hat{v} \frac{\partial \delta p}{\partial y}\right) d \Omega d t=0
$$

for arbitrary variations $\delta p$ in the pressure. Applying Gauss' theorem then yields that

$$
\alpha_{2} \int_{T_{a}}^{T_{b}} \int_{\Gamma_{s}} \delta p\left(\tau_{2}-\tau_{b}\right) d \Gamma-\int_{0}^{T} \int_{\Omega} \delta p\left(\frac{\partial \hat{u}}{\partial x}+\frac{\partial \hat{v}}{\partial y}\right) d \Omega d t+\int_{0}^{T} \int_{\Gamma} \delta p\left(\hat{u} n_{1}+\hat{v} n_{2}\right) d \Gamma d t=0
$$

where $n_{1}$ and $n_{2}$ denote the $x$ and $y$ components $\Gamma$ respectively $\Gamma$ of the outward normal to $\Omega$ along $\Gamma$. Choosing variations $\delta p$ that vanish on the boundary $\Gamma$ but which are arbitrary in the interior $\Omega$ of the flow domain yields that

$$
\frac{\partial \hat{u}}{\partial x}+\frac{\partial \hat{v}}{\partial y}=0 \quad \text { on }(0, T) \times \Omega
$$

Now choosing variations $\delta p$ that are arbitrary along the boundary $\Gamma$ yields that

$$
\hat{u} n_{1}+\hat{v} n_{2}=\left\{\begin{array}{l}
0 \quad \text { on }(0, T) \times \Gamma \backslash \Gamma_{s},\left(0, T_{a}\right) \times \Gamma_{s},\left(T_{b}, T\right) \times \Gamma_{s} \\
-\alpha_{2}\left(-p+2 \nu \frac{\partial v}{\partial y}-\tau_{b}\right) \quad \text { on }\left(T_{a}, T_{b}\right) \times \Gamma_{s},
\end{array}\right.
$$


where $\Gamma \backslash \Gamma_{s}$ denotes the boundary $\Gamma$ with $\Gamma_{s}$ deleted. Note that in the above derivation of (13) and (14) Tas in the derivations found below $\Gamma$ the boundary integrals at infinity do not make any contribution due to the last relation in (12).

Next $\Gamma$ consider $\delta \mathcal{L} / \delta v=0$ where equation (12) has been used to eliminate boundary integrals along $\Gamma_{i} \Gamma \Gamma_{w}$ and as $x \rightarrow \infty$ and an integral over $\Omega$ at $t=0$. First $\Gamma$ variations $\delta v$ that vanish at $t=0 \Gamma t=T \Gamma$ and in a neighborhood of $\Gamma$ are chosen $\Gamma$ but which are otherwise arbitrary. Such a choice implies that all boundary integrals in (12) vanish giving

$$
\begin{aligned}
-\frac{\partial \hat{v}}{\partial t}+\hat{u} \frac{\partial u}{\partial y}+\hat{v} \frac{\partial v}{\partial y}-u \frac{\partial \hat{v}}{\partial x}-v \frac{\partial \hat{v}}{\partial y}-\frac{\partial \hat{p}}{\partial y} & \\
& -\nu \frac{\partial}{\partial x}\left(\frac{\partial \hat{u}}{\partial y}+\frac{\partial \hat{v}}{\partial x}\right)-\nu \frac{\partial}{\partial y}\left(2 \frac{\partial \hat{v}}{\partial y}\right)=0 \quad \text { in }(0, T) \times \Omega,
\end{aligned}
$$

where equation (3) is used to effect a simplification. Next $\Gamma$ variations that vanish in a neighborhood of $\Gamma \Gamma$ but which are otherwise arbitrary $\Gamma$ are chosen to obtain

$$
\left.\hat{v}\right|_{t=T}=0 \text { in } \Omega
$$

Now $\Gamma$ along $\Gamma \Gamma \delta v$ and $\partial \delta v / \partial n$ may be independently selected $\Gamma$ provided that (12) is satisfied $\Gamma$ where $\partial / \partial n$ denotes the derivative in the direction of the outward normal to $\Omega$ along $\Gamma$. If $\delta v=0$ and $\partial \delta v / \partial n$ varies arbitrarily along $\Gamma \Gamma$ then

$$
\hat{v}=\left\{\begin{array}{cl}
0 & \text { on }(0, T) \times \Gamma \backslash \Gamma_{s},\left(0, T_{a}\right) \times \Gamma_{s},\left(T_{b}, T\right) \times \Gamma_{s} \\
\alpha_{2}\left(-p+2 \nu \frac{\partial v}{\partial y}-\tau_{b}\right) & \text { on }\left(T_{a}, T_{b}\right) \times \Gamma_{s} .
\end{array}\right.
$$

To see this $\Gamma$ note that along the inflow $\Gamma \Gamma_{i} \Gamma n_{2}=0$ and $\partial / \partial n=-\partial / \partial x$ while along the top and bottom boundaries $n_{1}=0 \Gamma \partial / \partial n= \pm \partial / \partial y$ Trespectively $\Gamma$ and $\Gamma$ since $\delta v=0 \Gamma \partial \delta v / \partial x=$ 0 . Note that (14) and (17) agree on the boundary segments where they simultaneously apply. Finally $\Gamma \delta$ is arbitrarily chosen along $\Gamma_{a}$ to obtain

$$
s_{2}=-\hat{p} n_{2}-\hat{v}\left(u n_{1}+v n_{2}\right)-\nu\left(\frac{\partial \hat{u}}{\partial y}+\frac{\partial \hat{v}}{\partial x}\right) n_{1}-2 \nu \frac{\partial \hat{v}}{\partial y} n_{2} \quad \text { on } \quad(0, T) \times \Gamma_{a} .
$$

Next $\Gamma$ consider $\delta \mathcal{L} / \delta u=0$. Applying to the resulting equation the same process that led to (15)-(18) yields

$$
\begin{aligned}
&-\frac{\partial \hat{u}}{\partial t}+\hat{u} \frac{\partial u}{\partial x}+\hat{v} \frac{\partial v}{\partial x}-u \frac{\partial \hat{u}}{\partial x}-v \frac{\partial \hat{u}}{\partial y}-\frac{\partial \hat{p}}{\partial x} \\
&-\nu \frac{\partial}{\partial x}\left(2 \frac{\partial \hat{u}}{\partial x}\right)-\nu \frac{\partial}{\partial y}\left(\frac{\partial \hat{u}}{\partial y}+\frac{\partial \hat{v}}{\partial x}\right)=0 \quad \text { in }(0, T) \times \Omega .
\end{aligned}
$$




$$
\hat{u}=\left\{\begin{array}{c}
\left.\hat{u}\right|_{t=T}=0 \quad \text { in } \Omega, \\
0 \quad \text { on }(0, T) \times \Gamma \backslash \Gamma_{s},\left(0, T_{a}\right) \times \Gamma_{s},\left(T_{b}, T\right) \times \Gamma_{s} \\
\alpha_{1}\left(\nu \frac{\partial u}{\partial y}-\tau_{a}\right) \quad \text { on }\left(T_{a}, T_{b}\right) \times \Gamma_{s},
\end{array}\right.
$$

and

$$
s_{1}=-\hat{p} n_{1}-\hat{u}\left(u n_{1}+v n_{2}\right)-2 \nu \frac{\partial \hat{u}}{\partial x} n_{1}-\nu\left(\frac{\partial \hat{u}}{\partial y}+\frac{\partial \hat{v}}{\partial x}\right) n_{2} \quad \text { on } \quad(0, T) \times \Gamma_{a} .
$$

In deriving (21) we have used the assumption that $\Gamma_{s}$ is part of the lower boundary of the channel so that along $\Gamma_{s}$ we have that $n_{2}=-1$. Again $\Gamma$ there is no conflict between (14) and (21) along boundary segments on which both apply.

\subsection{The Optimality Conditions}

The only first-order necessary conditions left to consider are $\delta \mathcal{L} / \delta g_{1}=0$ and $\delta \mathcal{L} / \delta g_{2}=$ 0. (These conditions are usually called the optimality conditions.) Now $\Gamma$ since all candidate functions $g_{1}$ and $g_{2}$ must statisfy (9) $\Gamma$ it follows that $\delta g_{1}=0$ and $\delta g_{2}=0$ at $t=T_{0}$. Then $\Gamma$ take $\delta \mathcal{L} / \delta g_{2}=0$ and apply Gauss' theorem to remove all derivatives from the variation $\delta g_{2}$. Choosing variations $\delta g_{2}$ that vanish at $t=T_{1}$ Tbut which are otherwise arbitraryTand using (18) yields

$$
-\frac{\partial^{2} g_{2}}{\partial t^{2}}+g_{2}=-\frac{1}{\beta_{2}}\left(\hat{p}+2 \nu \frac{\partial \hat{v}}{\partial y}\right) \text { on }\left(T_{0}, T_{1}\right) \times \Gamma_{a}
$$

where (17) and the assumption that $\Gamma_{a}$ is part of the lower boundary so that $\Gamma$ along $\Gamma_{a} \Gamma$ $n_{1}=0$ and $n_{2}=-1$ have been used. Now choosing variations that are arbitrary at $t=T_{1}$ yields that $\partial g_{2} / \partial t=0$ along $\Gamma_{a}$ at $t=T_{1}$ so that $\Gamma$ invoking $(9) \Gamma g_{2}(t, x)$ satisfies

$$
\left.g_{2}\right|_{t=T_{0}}=g_{20}(x) \quad \text { and }\left.\quad \frac{\partial g_{2}}{\partial t}\right|_{t=T_{1}}=0 \quad \text { on } \Gamma_{a}
$$

Note that $\Gamma$ given $\hat{p}$ and $\hat{v} \Gamma(23)$ and (24) constitute $\Gamma$ at each point $x \in \Gamma_{a} \Gamma$ a two-point boundary value problem in time over the interval $\left(T_{0}, T_{1}\right)$.

In a similar manner $\Gamma$ setting $\delta \mathcal{L} / \delta g_{1}=0$ yields that

$$
\begin{gathered}
-\frac{\partial^{2} g_{1}}{\partial t^{2}}+g_{1}=-\frac{1}{\beta_{1}}\left(\nu \frac{\partial \hat{u}}{\partial y}\right) \quad \text { on }\left(T_{0}, T_{1}\right) \times \Gamma_{a} \\
\left.g_{1}\right|_{t=T_{0}}=g_{10}(x) \quad \text { and }\left.\quad \frac{\partial g_{1}}{\partial t}\right|_{t=T_{1}}=0 \quad \text { on } \Gamma_{a} .
\end{gathered}
$$




\subsection{Finite Computational Domains}

In the computations $\Gamma$ the semi-infinte domain $\Omega$ (we are still only considering the channel flow case) is replaced by a finite domain $\Omega_{C}$ defined by the introduction of the outflow boundary $\Gamma_{o}$ given by $[x=L \Gamma 0 \leq y \leq h]$. Thus $\Gamma$ we have that $\Omega_{C}$ is the rectangle $[0 \leq x \leq L \Gamma 0 \leq y \leq h]$. The outflow does not require the imposition of boundary conditions along the outflow boundary $\Gamma_{o}$ because a buffer zone ${ }^{27}$ is attached to the end of the physical computational domain $\Gamma$ where the governing equations are parabolized in this buffer region.

A similar treatment of the adjoint variables should have required consideration of an infinite domain $[-\infty<x<\infty \Gamma 0<y<h]$. If this had been done $\Gamma$ the boundary conditions (17) and (21) would not have been obtained along the inflow $\Gamma_{i}$. In fact $\Gamma$ the inflow boundary $\Gamma_{i}$ for the state equation is the outflow boundary for the adjoint equations and $\Gamma$ conversely $\Gamma$ the outflow boundary $\Gamma_{o}$ for the state equation is the inflow boundary for the adjoint equations. This is easily seen by comparing the leading inertial terms of the state equations with $t$ increasing and the adjoint equations with $t$ decreasing. Now $\Gamma$ on both $\Gamma_{i}$ and $\Gamma_{o}$ we have that $u>0$ and $v \approx 0$ which is why $\Gamma_{i}$ is an inflow boundary and $\Gamma_{o}$ is an outflow boundary for the state. On the other hand $\Gamma$ the fact that $t$ is decreasing in the adjoint equations implies that now $\Gamma_{i}$ is an outflow boundary and $\Gamma_{o}$ is an inflow boundary for those equations.

Thus Tto be consistent with the treatment of the state equations $\Gamma$ the adjoint outflow $\Gamma_{i}$ should be treated in a manner similar to the above treatment of the state outflow $\Gamma_{o}$. This treatment of the adjoint outflow does not require the imposition of any boundary conditions for the adjoint variables along $\Gamma_{i}$. Finally $\Gamma_{\text {since }} \Gamma_{o}$ is an inflow boundary for the adjoint equations Tone has that

$$
\hat{u}=0 \quad \text { and } \quad \hat{v}=0 \quad \text { on }(0, T) \times \Gamma_{o} .
$$

\subsection{The Optimality System for Channel Flow}

We now have in hand the full optimality system for channel flow whose solutions determine the optimal states $\Gamma$ controls $\Gamma$ and adjoint states. These are:

$$
\begin{gathered}
\text { State equations }-(1)-(7) \\
\text { Co-state equations }-(13)-(17),(19)-(21), \text { (27) } \\
\text { Optimality equations - (23)-(26) }
\end{gathered}
$$

Since (18) and (22) merely serve to determine the uninteresting Lagrange multipliers $s_{2}$ and $s_{1} \Gamma$ they can be ignored. 
The state equations are driven by the given initial velocity $\left(u_{0}, v_{0}\right) \Gamma$ the given inflow velocity $\left(u_{i}, v_{i}\right)$ Tand the controls $\left(g_{1}, g_{2}\right)$. Indeed $\Gamma$ the purpose of this study is to determine $g_{1}$ and $g_{2}$ that optimally counteracts instabilities created upstream of $\Gamma_{a}$. The adjoint equations are homogeneous except for the boundary condition along $\Gamma_{s} \Gamma$ the part of the boundary along which we are trying to match the stresses. The data in that boundary condition is exactly the discrepancy between the desired stresses $\tau_{a}$ and $\tau_{b}$ and the stresses $\tau_{1}=\nu \partial u / \partial y$ and $\tau_{2}=-p+2 \nu \partial u / \partial y$ along $\Gamma_{s} \Gamma$ weighted by the factors $\alpha_{1}$ and $\alpha_{2}$. The equations for the controls are driven by the negative of the adjoint stresses along $\Gamma_{a} \Gamma$ the part of the boundary along which we apply the control weighted by the factors $1 / \beta_{1}$ and $1 / \beta_{2}$. Of course this division into equations for the state $\Gamma$ the adjoint state $\Gamma$ and the control is really obscured by the fact that equations are all intimitately coupled.

\subsection{The Optimality System for Boundary-Layer Flow}

Following a similar process to that used in Sections 3.1-3.5 for the channel flow case $\Gamma$ one may derive an optimality system for the boundary layer flow case. The only difference is that in the latter case $\Gamma_{w}$ denotes only the lower boundary with $\Gamma_{a}$ excluded and that the additional boundary condition (8) along the upper boundary $\Gamma_{e}$ must be accounted for.

With the new interpretation for $\Gamma_{w} \Gamma$ one can still define the Lagrangian functional (11) and use the constraints (12) on allowable variations; however $\Gamma$ due to (8) $\Gamma$ allowable variations are further constrained by

$$
\left.\delta u\right|_{\Gamma_{e}}=\left.\left(\delta p-2 \nu \frac{\partial \delta v}{\partial y}\right)\right|_{\Gamma_{e}}=0 \quad \text { for }(0, T) .
$$

which implies that $\Gamma$ along $\Gamma_{e}$ Tone may not choose the variations in $\delta p$ and $\partial \delta v / \partial y$ independently. Considering $\Gamma$ simultaneously $\Gamma$ variations in $p \Gamma v \Gamma$ and $\partial v / \partial y$ along $\Gamma_{e} \Gamma$ one can show that

$$
\hat{u}=0 \quad \text { on }(0, T) \times \Gamma_{e}
$$

Then letting $\delta v$ be arbitrary along $\Gamma_{e}$ yields

$$
\hat{p}+2 \nu \frac{\partial \hat{v}}{\partial y}+v \hat{v}=0 \quad \text { on }(0, T) \times \Gamma_{e} .
$$

The resulting system for boundary-layer flow now includes (28) and (29) in addition to the channel flow system. 


\section{Numerical Experiments}

Here the optimal control methodology developed in Section 3 is applied to a boundarylayer flow having a single instability wave that can be characterized by a discrete frequency within the spectrum. As described by Joslin et al. $I^{21,28}$ these discrete small-amplitude instabilities can be suppressed through wave cancellation (WC) using known information about the wave. Hence $\Gamma$ the optimal control is "known" for validation of the present DNS/optimal control theory numerical approach in which the instability is to be suppressed without any a priori knowledge of said instability.

We note that although we are testing our methodology and code for the special problem of two-dimensional TS wave suppression $\Gamma$ that these were developed for the fully nonlinear Navier-Stokes system and thus are applicable to the case of nonlinear $\Gamma$ threedimensional waves. We would expect that more iterations $\Gamma$ and perhaps a refined interation procedure $\Gamma$ would be needed for convergence in a multi-mode case. Also Twe have successfully suppressed a single large-amplitude TS wave; space limitations have prevented us from presenting these results here.

The formidable coupled system is solved in an iterative manner. First $\Gamma$ the NavierStokes equations are solved for the state variables $\Gamma$ i.e. $\Gamma$ the velocity field $(u, v)$ and pressure $p$ with control information (i.e. $\Gamma$ no control $g_{1}=g_{2}=0$ for first iteration). Then co-state equations are solved for the adjoint or co-state variables $(\hat{u}, \hat{v})$ and $\hat{p}$. Then $\Gamma$ using these adjoint variables $\Gamma$ the controls $g_{1}$ and $g_{2}$ are then found by solving the optimality equations. The procedure is repeated until satisfactory convergence is achieved.

The nonlinear $\Gamma$ unsteady Navier-Stokes equations and linear adjoint Navier-Stokes equations are solved by direct numerical simulation (DNS) of disturbances that evolve spatially within the boundary layer. The spatial $\mathrm{DNS}^{29,30}$ approach involves spectral and high-order finite-difference methods and a three-stage Runge-Kutta method ${ }^{31}$ for time advancement. The influence-matrix technique is employed to solve the resulting pressure equation. ${ }^{20,32}$ Disturbances are forced into the boundary layer by unsteady suction and blowing through a slot in the wall. The buffer-domain technique ${ }^{27}$ is used for the outflow boundary treatment.

In the present study only normal injection or suction control is allowed $\Gamma$ so that we set $g_{1}=0$ in $(5) \Gamma \beta_{1}=0$ in the functional (10) $\Gamma$ and ignore (25) and (26). Also $\Gamma$ we only match the normal stress along $\Gamma_{s}$ so that we choose $\alpha_{1}=0$ in the functional (10) and in $(21)$. 


\subsection{Computational Parameters}

For the computations $\Gamma$ the grid has 401 streamwise and 41 wall-normal points. The free stream boundary is located $75 \delta_{o}^{*}$ from the wall $\Gamma$ and the streamwise length is $224 \delta_{o}^{*}$ which is equal to approximately 8 TS wavelengths. The nondimensional frequency for the forced disturbance is $F=\omega / R \times 10^{6}=86$; the forcing amplitude is $v_{f}=0.1 \%$. The Reynolds number based on the inflow displacement thickness $\left(\delta_{o}^{*}\right)$ is $R=900$. (The boundary segment along which disturbance forcing and control is effected as well as where stress matching occurs are located within the unstable region of the linear stability neutral curve.) A time-step size corresponding to 320 steps per period $T_{p}$ is chosen for a threestage Runge-Kutta method. Based on the disturbance frequency $\Gamma$ a characteristic period can be defined as $T_{p}=2 \pi / \omega=81.1781$; the resulting time-step size is then $\Delta t=0.2537$.

To complete one period of the active-control simulation process $\Gamma 0.75$ minutes on the Cray C-90 are required using a single processor. Note $\Gamma$ two periods of cost $\left(T_{a} \rightarrow T_{b}\right.$ and $T_{b} \rightarrow T_{a}$ ) are required to complete one iteration of the DNS/adjoint system. Although in general any time interval may be specified for $T_{a} \rightarrow T_{b} \Gamma$ this study uses integer increments of the period $\left(T_{p}\right)$ for simplicity. Hence $\Gamma T_{a} \rightarrow T_{b}=2 T_{p}$ would cost $4 T_{p}$ in computations $\Gamma$ or roughly $3 \mathrm{~min}$ of $\mathrm{C}-90$ time per iteration. Because only a single small-amplitude wave (linear) is forced $\Gamma$ the above grid is more than adequate; however $\Gamma$ a grid refinement was performed and produced results equivalent to the results reported here.

For this study $\Gamma$ the disturbance forcing slot $\Gamma_{f} \Gamma$ the control or actuator orifice $\Gamma_{a} \Gamma$ and the matching or sensor segment $\Gamma_{s}$ have equal length $4.48 \delta_{o}^{*}$. The forcing is centered downstream at $389.62 \delta_{o}^{*}$ (the Reynolds number based on the displacement thickness at that location is $R=1018.99) \Gamma$ the actuator is centered at $403.62 \delta_{o}^{*}(R=1037.13) \Gamma$ and the sensor is centered at $417.62 \delta_{o}^{*}(R=1054.97)$. These separation distances were arbitrarily chosen for this demonstration. In practice $\Gamma$ the control and matching segments should have a minimal separation distance so that the pair can be packaged as a single unit for distributed application of many bundles.

\subsection{Results}

All simulations allow the flow field to develop for one period $\Gamma$ i.e. Ifrom $t=0 \rightarrow T_{a}=T_{p}$ before control is initiated. In the first series of simulations $\Gamma$ the interval during which control is applied is arbitrarily chosen to be $T_{a} \rightarrow T_{b}=2 T_{p}$. Based on $\alpha_{1}=\beta_{1}=0 \Gamma \alpha_{2}=1 \Gamma$ and $\beta_{2}=10 \Gamma$ the convergence history for the wall-normal velocity and measured normal shear $\tau_{2}$ are shown in Fig. 2. The velocities are obtained at a fixed distance from the wall corresponding to $1.18 \delta_{o}^{*}$ and at the fixed time $T_{b}$. Convergence is obtained with 4 
iterations. The results demonstrate that a measure of wave cancellation can be obtained from the DNS/control theory system. The wall-normal amplitude of the modified wave at $R=1092.5$ is 40 percent of the uncontrolled wave; the control without optimizing the choice of $\alpha_{1} \Gamma \alpha_{2} \Gamma \beta_{1} \Gamma$ and $\beta_{2}$ has led to a 60 percent decrease in the amplitude of the travelling wave. Clearly FFig. 2 shows that a net reduction of the disturbance energy is obtained by energy input due to the control. This results in a delay of transition by-way-of a suppression of the instability evolution.

In the simulation $\Gamma$ the control has been applied from $T_{a} \rightarrow T_{b}$ only; therefore $\Gamma$ for $t>T_{b}$ Tequation (5) indicates that the actuation is discontinued. Figure 3 compares velocity profiles at $R=1073.2$ for the converged results (C1) of Fig. 2 with results for one period after control $t=T_{b}+T_{p}$. The measured disturbance tends toward the uncontrolled solution when the actuation is discontinued (as expected); because the control was applied for $T_{a}-T_{p} \rightarrow T_{b}=2 T_{p} \Gamma 2$ periods are required after $T_{b}$ before the computed solution in the window exactly matches the uncontrolled solution. Clearly $\Gamma$ the control only removes energy from the system (decreases the wave instability amplitude); the resulting profiles retain the expected instability profile shape.

The effect of varying the window size $\left(T_{a}, T_{b}\right)$ is demonstrated. The previous converged results $(\mathrm{C} 1)$ are shown with converged results $(\mathrm{C} 2)$ for the extended window $\left(T_{a}=T_{p} \rightarrow\right.$ $\left.T_{b}=3 T_{p}\right)$. The results are identical for the first two periods of time and indicate that extending the amount of time for control serves to extend control only. This result also indicates that one can solve for the optimal control over a given time interval $\left(T_{a}, T_{b}\right)$ by breaking up that inerval and solving for the optimal control over a series of smaller subintervals. This approach usually leads to substantial savings in CPU and memory costs. An additional insight about the present DNS/control theory is gleamed by the increased temporal frame. The resulting optimal control $g_{2}$ approaches the desired wavecancellation time-periodic solution as the temporal length $\left(T_{a}, T_{b}\right)$ is increased. This is convincing evidence that the present self-contained methodology is valid.

The instability wave resulting from wave-cancellation (WC) is shown with the control (C2) in Figs. 4 and 5. For the present comparison Tthe amplitude of the actuation for WC was adjusted until nearly exact wave cancellation was achieved. Although the DNS/control theory did not achieve the same level of energy removal $\Gamma$ the similar effect of WC was achieved without any a priori knowledge of the instability. AlsoTnote that Fig. 5 shows that the optimal control of the control theory has nearly the exact phase characteristics as WC and only lacks the necessary amplitude for additional wave cancellation. These encouraging results suggest that by the appropriate selection of $\alpha_{1} \Gamma \alpha_{2} \Gamma \beta_{1} \Gamma$ and $\beta_{2} \Gamma$ the 
optimal control can be made nearly as effective method of instability suppression as exact wave cancellation.

From the wave-cancellation study of Joslin et al.I ${ }^{21}$ the relationship between amplitude of the actuator $\left(v_{a}\right)$ with resulting instabilty can be shown in Fig. 6. A similar result was shown in the channel flow wave-cancellation study in Biringen. ${ }^{14}$ The trend indicates $\Gamma$ that beginning with a small actuation amplitude $\Gamma$ as the actuation level is increased $\Gamma$ the amount of wave cancellation (energy extraction from the disturbance) increases. At some optimal actuation $\Gamma$ nearly exact wave cancellation is achieved for the instability wave. As the actuation amplitude further increases the resulting instability amplitude increases; this was clearly explained in Joslin et al. ${ }^{21}$ to occur because in the wave superposition process $\Gamma$ the actuator wave becomes dominant over the forced wave. At this point $\Gamma$ the resulting instability undergoes a phase shift corresponding to the phase of the wave generated by the actuator. The relationship depicted in Fig. 6 is encouraging for the DNS/optimal control theory approach and suggests that a gradient descent type algorithm might further enhance the wave suppression capability of the present approach. Namely $\Gamma$ an approach for the optimal selection of $\alpha_{1} \Gamma \alpha_{2} \Gamma \beta_{1} \Gamma$ and $\beta_{2}$ might lead to a more useful theoretical/computational tool for flow control.

To simply demonstrate this concept $\Gamma$ Lagrange interpolation (or perhaps extrapolation) is introduced for $\beta_{1}$ and $\beta_{2}$ based on imposed values for $\alpha_{1}$ and $\alpha_{2}$ :

$$
\beta_{1,2}^{n+1}=\frac{\beta_{1,2}^{n}\left(\tau_{1,2}^{*}-\tau_{1,2}^{n-1}\right)-\beta_{1,2}^{n-1}\left(\tau_{1,2}^{*}-\tau_{1,2}^{n}\right)}{\left(\tau_{1,2}^{n}-\tau_{1,2}^{n-1}\right)},
$$

where $\tau_{1,2}^{*}$ are some desired values of the stress components and $\tau_{1,2}^{n}$ are the stress components based on the choice $\beta_{1,2}^{n}$. Although $\tau_{1}^{*}$ and $\tau_{2}^{*}$ may be equivalent to the target values $\tau_{a}$ and $\tau_{b}$ in the functional (10) $\Gamma$ this may lead to significant over/under shoots for the interation process. Instead $\Gamma \tau_{1}^{*}$ and $\tau_{2}^{*}$ is the incremental decrease $\Gamma$ or target value $\Gamma$ for interpolation to more desirable $\beta_{1}$ and $\beta_{2}$ values. To illustrate this process $\Gamma$ the $\beta_{2}=10$ $(\mathrm{C} 2)$ and $\beta_{2}=11$ control results are obtained with the iteration procedure. The measures of normal stress are somewhat arbitrarly obtained at some time as measured by the sensor or matching segment $\Gamma_{s}$; the values of the normal stress are given in the Table 1 . These values are used for a desired normal stress $\tau_{2}^{*} \Gamma$ which in this case is $65 \%$ of the $\beta_{2}=11$ results.

Table 1. Normal stress for two values of $\beta_{2}$.

\begin{tabular}{|c|c|}
\hline$\beta_{2}$ & normal stress \\
\hline 10 & $9.369 \times 10^{-6}$ \\
11 & $8.814 \times 10^{-6}$ \\
\hline
\end{tabular}


Using the results for $\beta_{2}=10$ and $\beta_{2}=11$ in (30) yields the value $\beta_{2}=16.5$ which is used in a simulation to obtain a greater degree of instability suppression. The WC results and the enhanced optimal control (C3) solution are shown in Figs. 7 and 8 . This interpolation approach based on relationship of Fig. 6 indicates that optimizing $\beta_{2}$ has led to results very close to $\mathrm{WC}$. The solutions differ somewhat near $t=T_{a}$ and $t=T_{b}$ because of the conditions (24) and (26) that serve to control the levels of $g_{1}$ and $g_{2}$. For all practical purposes Tthe solutions obtained with the present DNS/control theory methodology yield the desired flow control features without prior knowledge of the forced instability.

The adjoint system requires that the velocity field $(u, v)$ obtained from the NavierStokes equations be known for all time. For the iteration sequence and a modestly course gridГ82 Mbytes of disk (or runtime) space are required to store the velocities at all time steps and for all grid points. For $T_{a} \rightarrow T_{b}=3 T_{p} \Gamma 246$ Mbytes are necessary for the computation. Clearly for three-dimensional problems the control scheme becomes prohibitively expensive. Therefore $\Gamma$ a secondary goal of this study is to determine if this limitation can be elliminated.

Because the characteristics of the actuator $\left(g_{1}\right.$ and $\left.g_{2}\right)$ and resulting solutions are comparable to WCT some focus should be placed on elliminating the enormous memory requirements discussed above. This limitation can easily be removed if the flow-control problem involves small-amplitude unsteadiness (or instabilities). The time-dependent coefficients of the adjoint system (15) and (19) reduce to the steady-state solution and no additional memory is required over the Navier-Stokes system in terms of coefficients. This has been verified by a comparison of a simulation with steady coefficients compared with the $\mathrm{C} 2$ control case. The results for both cases are identical (as expected). Additionally if the instabilities have small amplitudesTthen a linear Navier-Stokes solver can be used instead of the full nonlinear solver which was used in the present study. This linear system would be very useful for the design of flow-control systems. However $\Gamma$ if the instabilities in the flow have sufficient amplitude to interact nonlinearly $\Gamma$ then some measure of unsteady coefficient behavier is likely required. Depending on the amplitudes $\Gamma$ the coefficients saved at every time-step may be replaced with storing coefficients every 10 or more time-steps thereby reducing the memory requirements by an order of magnitude. This hypothesis will require validation in a future study.

\section{Conclusions}

The coupled Navier-Stokes equations $\Gamma$ adjoint Navier-Stokes $\Gamma$ and optimality condition equations were solved and validated for the flow-control problem of instability wave 
suppression in a flat plate boundary layer. By solving the above system $\Gamma$ optimal controls were determined that met the objective of minimizing the perturbation normal stress along a portion of the bounding wall. As a result $\Gamma$ the optimal control was found to be an effective means for suppressing two-dimensional unstable Tollmien-Schlichting travelling waves. The results indicate that the DNS/control theory solution is comparable to the wave-cancellation result but $\Gamma$ unlike the latter $\Gamma$ requires no a priori knowledge of the instability characteristics.

\section{Acknowledgements}

This research was supported by NASA under contract no. NAS1-19480 while the authors (except the first author) were in residence at the Institute for Computer Appli-

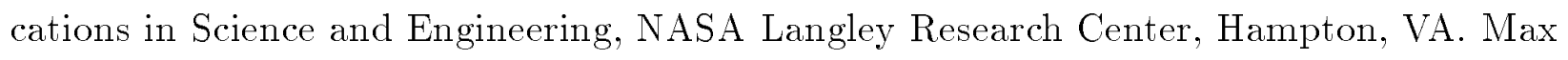
Gunzburger was also supported by the Air Force Office of Scientific Research under grant number AFOSR-93-1-0280.

\section{References}

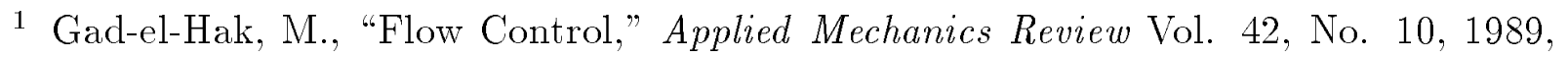
pp. 261-293.

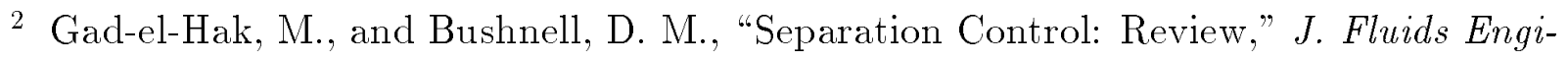
neering $\Gamma$ Vol. $113 \Gamma 1991 \Gamma$ pp. 5-30.

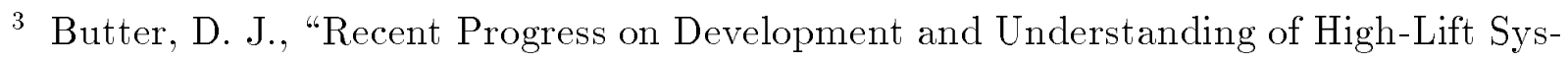
temsए AGARD-CP-365Г1984.

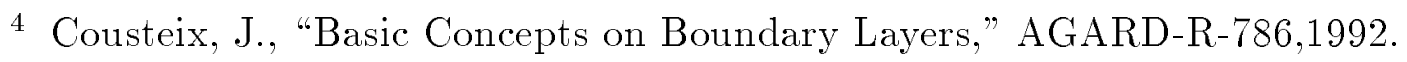

5 Muirhead TV. U. “"An Investigation of Drag Reduction for Tractor-Trailer Vehicles. NASA CR 144877 Г1978.

${ }^{6}$ LaddГD. M. and Hendricks $\Gamma E$. W. “ "Active Control of 2-D Instability Waves on an Axisymmetric Body ${ }^{\top}$ Experiments in Fluids Vol. 6Г1988Гpp. 69-70.

7 Pupator ГP. and Saric ГW. "Control of Random Disturbances in a Boundary LayerT" AIAA Paper 89-1007Г1989.

8 Ladd Г D. М. "Control of Natural Laminar Instability Waves on an Axisymmetric

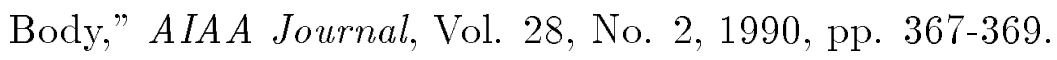

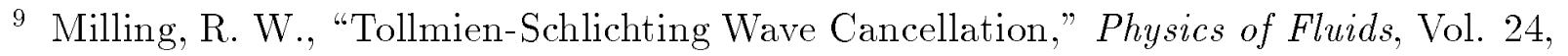
No. 5Г1981Грp. 979-981.

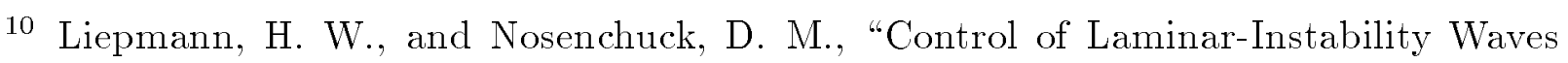

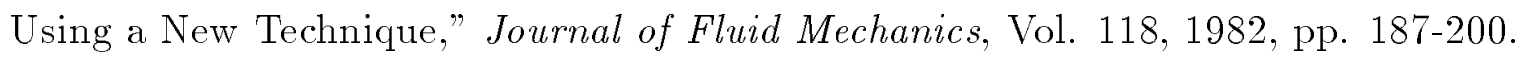




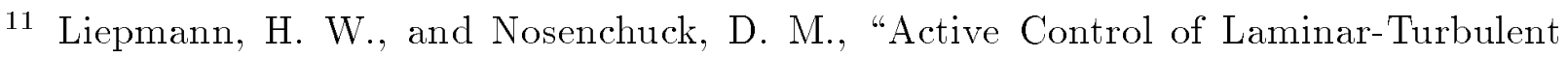
TransitionT Journal of Fluid Mechanics ГVol. 118Г1982Гpp. 201-204.

12 Thomas A. S. W. $\Gamma$ "The Control of Boundary-Layer Transition using a WaveSuperposition principleГ” Journal of Fluid Mechanics ГVol. 137Г1983Гpp.233-250.

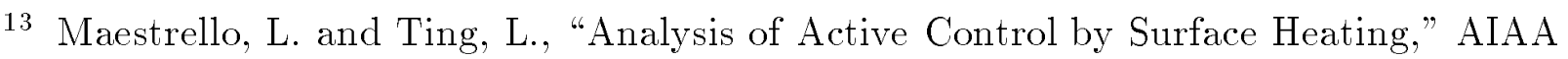
Paper 84-0173Г1984.

14 BiringenTS.T"Active Control of Transition by Periodic Suction-BlowingT" Physics of Fluids ГVol. 27ГNo. 6 Г1984Грp. 1345-1347.

15 MetcalfeГR. W.ГRutlandГС.ГDuncanГJ. Н.Гand RileyГJ. J. “Numerical Simulations of Active Stabilization of Laminar Boundary LayersT AIAA Paper 85-0567Г1985.

16 BowerГW. W.ГKegelmanГJ. Т.ГРа1ГА.Гand MeyerГG. Н.Г"А Numerical Study of Two-Dimensional Instability-Wave Control Based on the Orr-Sommerfeld EquationT Physics of Fluids Vol. 30ГNo. 4Г1987Гpp. 998-1004.

17 PalГ А.Г BowerГW. W. \& MeyerГG. H.Г "Numerical Simulations of Multifrequency Instability-Wave Growth and Suppression in the Blasius Boundary Layer ${ }^{\text {M Physics of }}$

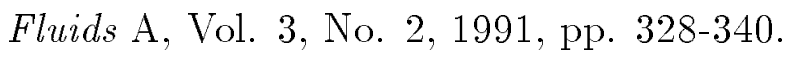

18 LaurienTE. and KleiserTL. "Numerical Simulation of Boundary-Layer Transition and Transition ControlГ J. Fluid Mech. Vol. 199Г1989Гpp. 403-440.

19 KralГL. D. and FaselГH. F. "Numerical Investigation of the Control of the Secondary Instability Process in Boundary LayersT AIAA Paper 89-0984Г1989.

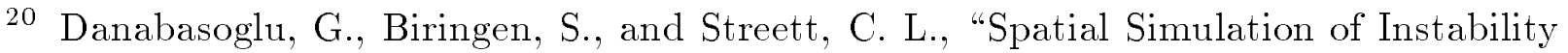
Control by Periodic Suction and BlowingT Physics of Fluids AГVol. 3ГNo. 9Г $1991 \Gamma$ pp. 2138-2147.

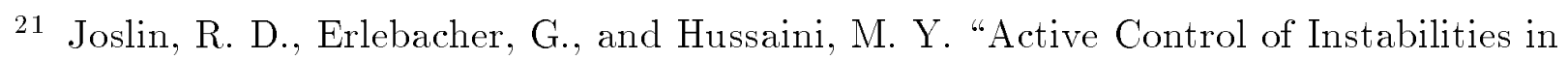
Laminar Boundary-Layer Flow. An OverviewT NASA CR 195016 ГICASE Report No. 94-97ГDecember 1994.

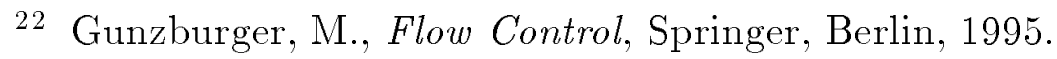

23 BorggaardГJ.ГBurkardtГ.Ј.ГGunzburgerГМ.Гand PetersonГJ.Г Optimal Design and Control $\Gamma$ Birkhauser $\Gamma$ Boston $\Gamma 1995$.

24 FursikovГА. V.ГGunzburgerГМ.Гand HouГL.Г“Boundary Value Problems and Optimal Boundary Control of the Navier-Stokes system: The Two-Dimensional CaseT to appear in SIAM J. Cont. Optim. 1996.

25 ChoiГН.ГTemamГR.ГМoinГР.Гand KimГJ.Г“Feedback Control of Unsteady Flow and its Application to the Stochastic Burgers EquationT Journal of Fluid Mechanics TVol. $253 \Gamma 1993 \Gamma$ рр. $509-543$. 
26 BewleyГТГМoinГР.Гаnd TemamГR.Г“А Method for Optimizing Feedback Control Rules for Wall Bounded Turbulent Flows Based on Control TheoryT Forum on Control

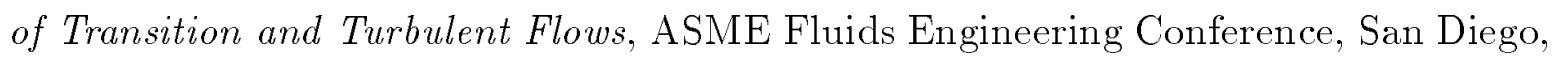
July 1996.

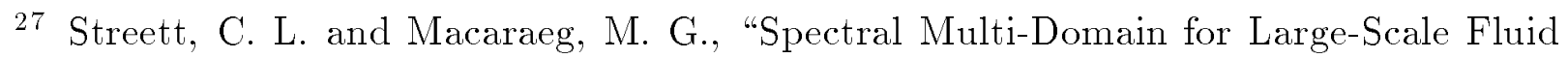
Dynamics SimulationsT Applied Numerical Mathematics TVol. 6Г1989Гpp. 123-140.

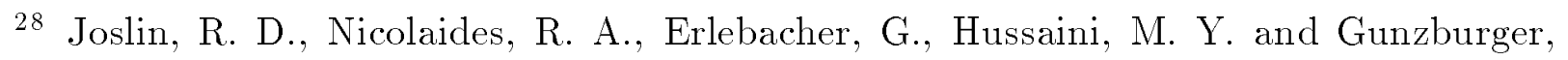
M. $\Gamma$ “Active Control of Boundary-Layer Instabilities: Use of Sensors and Spectral

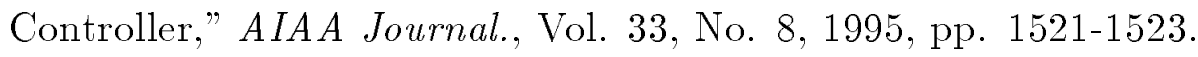

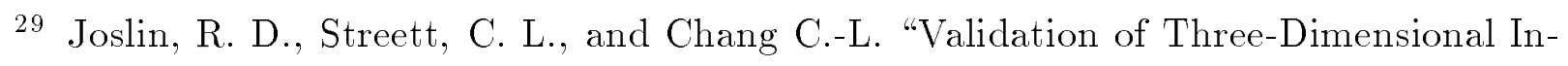
compressible Spatial Direct Numerical Simulation Code - A Comparison with Linear Stability and Parabolic Stability Equations Theories for Boundary-Layer Transition on a Flat PlateT NASA TP-3205ГJuly 1992.

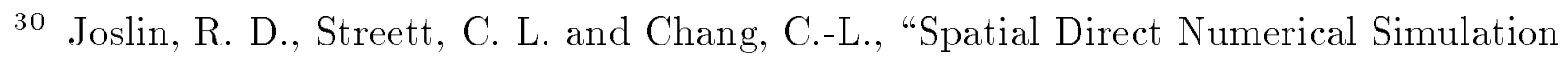
of Boundary-Layer Transition Mechanisms: Validation of PSE Theory $\mathrm{T}$ Theoretical

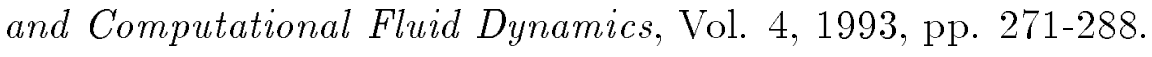

31 WilliamsonГJ. H.Г "Low-Storage Runge-Kutta SchemesT" Journal of Computational

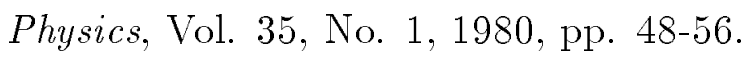

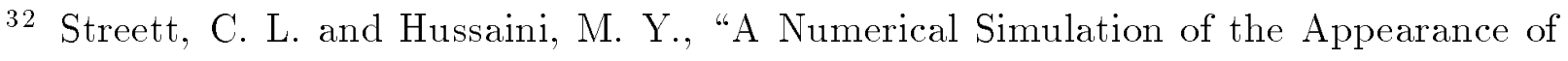
Chaos in Finite-Length Taylor-Couette Flow $\$$ Applied Numerical Mathematics $\Gamma$ Vol. $7 \Gamma 1991 \Gamma$ рp. $41-71$. 


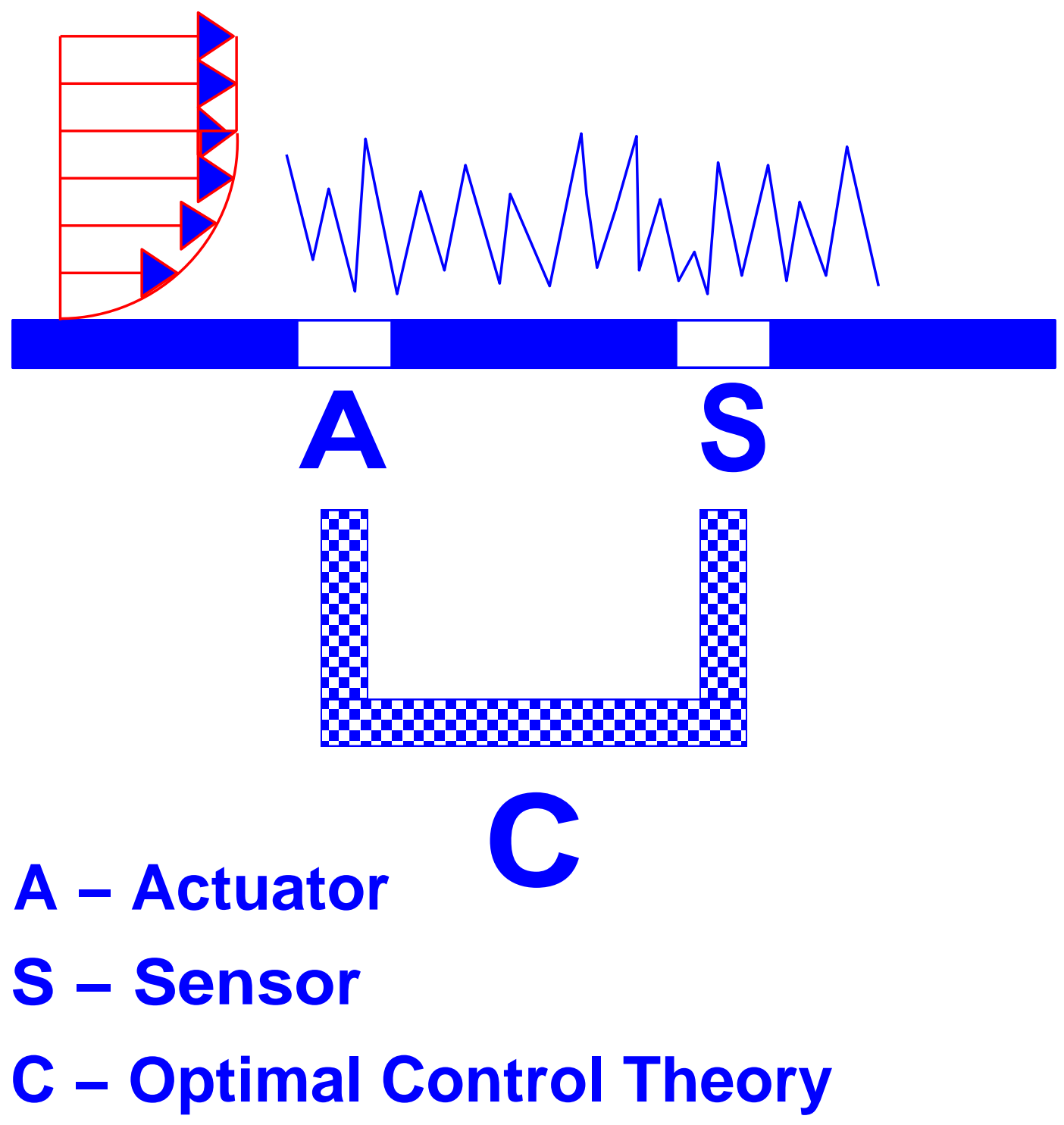

Fig. 1. Schematic of active flow control using optimal control theory. 

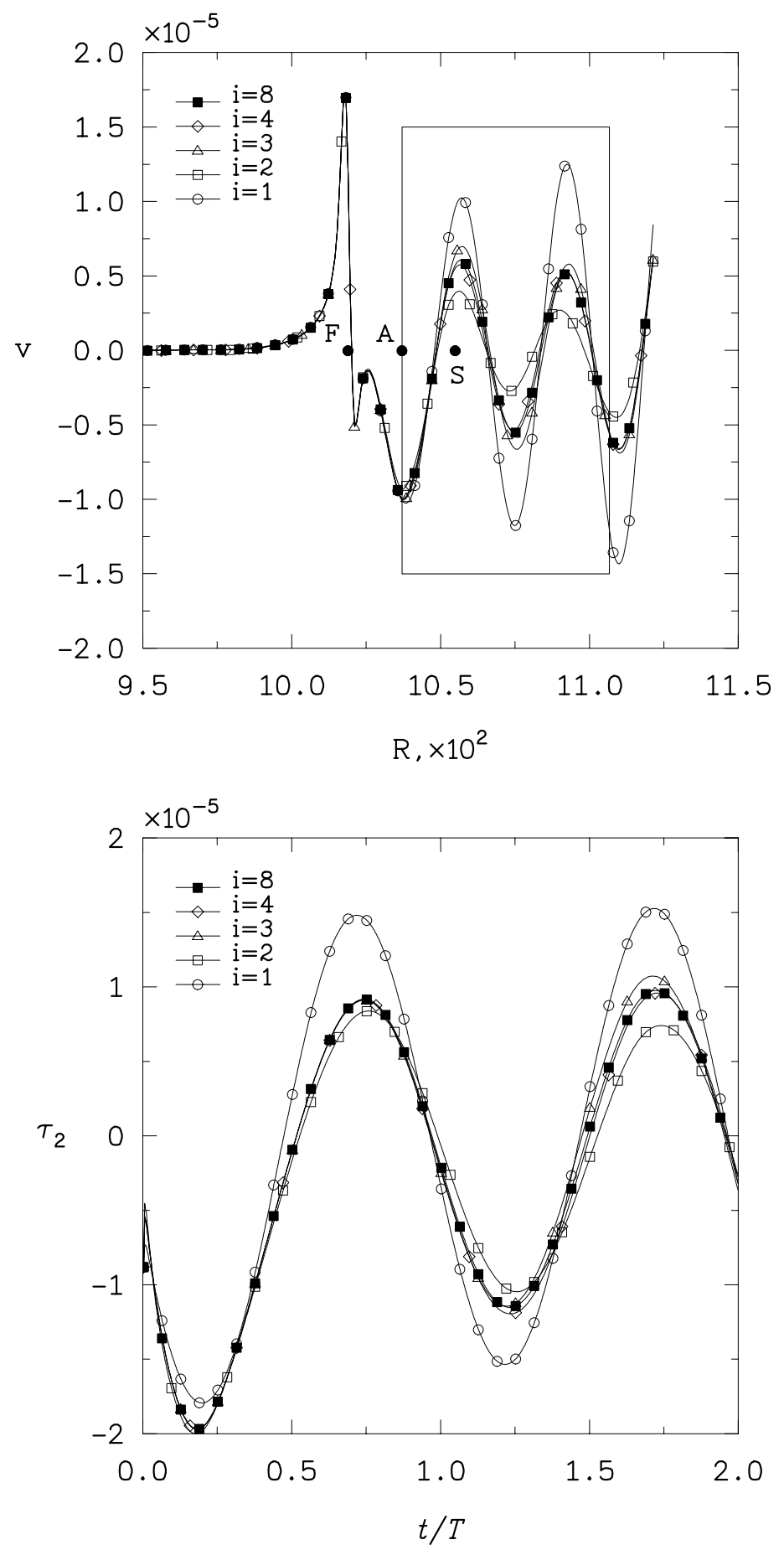

Fig. 2. Convergence of disturbance wall-normal velocity with downstream distance and sensor-measured shear stress with discrete time for control in flat-plate boundary-layer flow. (Velocity signal at $y=1.18 \delta_{o}^{*}$ from wall; $T_{1}-T_{0}=2 T_{p}$.) 

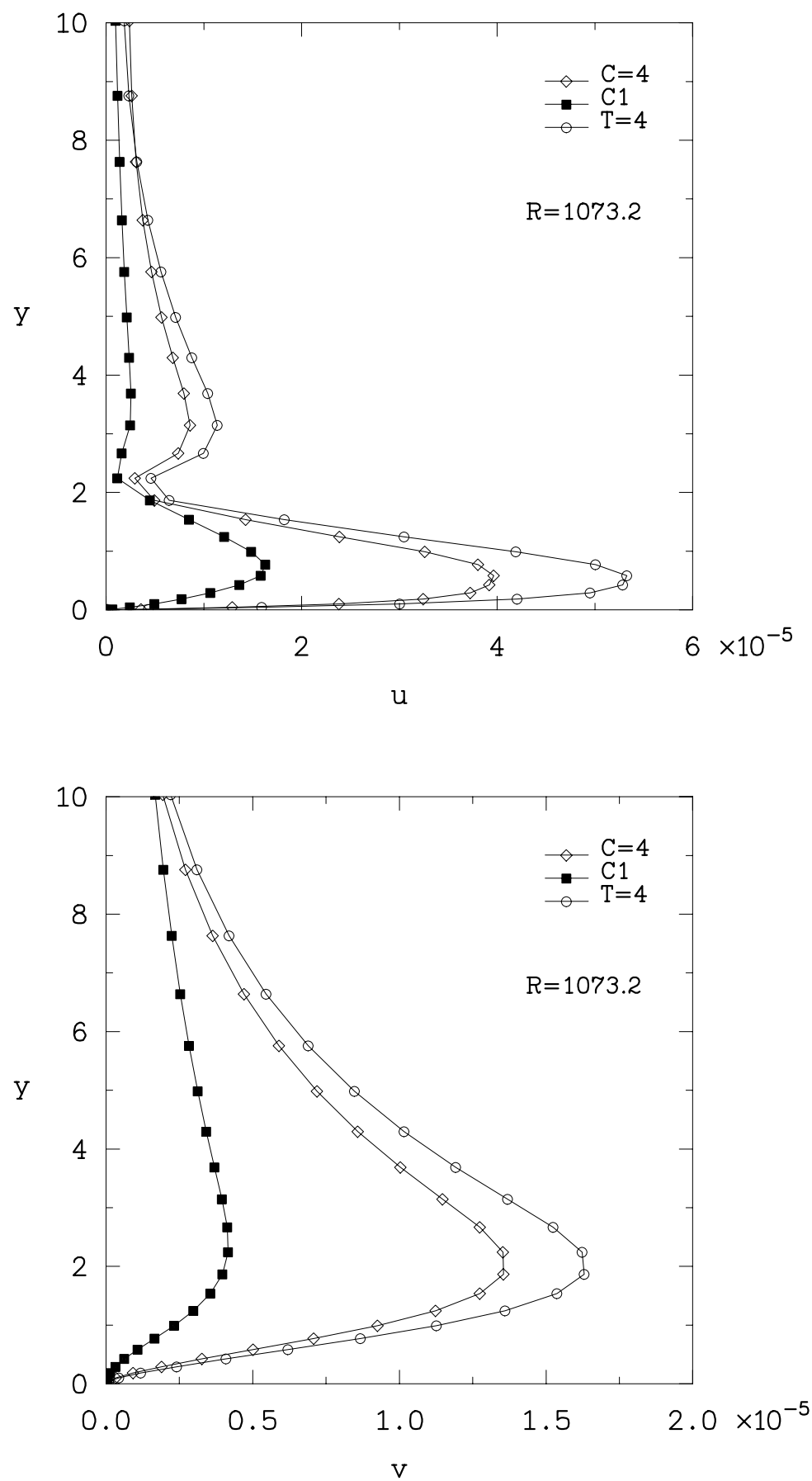

Fig. 3. Disturbance velocity profiles for no control $(\mathrm{T}=4) \Gamma \operatorname{control}(\mathrm{C} 1) \Gamma$ and after control is used and turned-off $(\mathrm{C}=4)$ in flat-plate boundary-layer flow. 


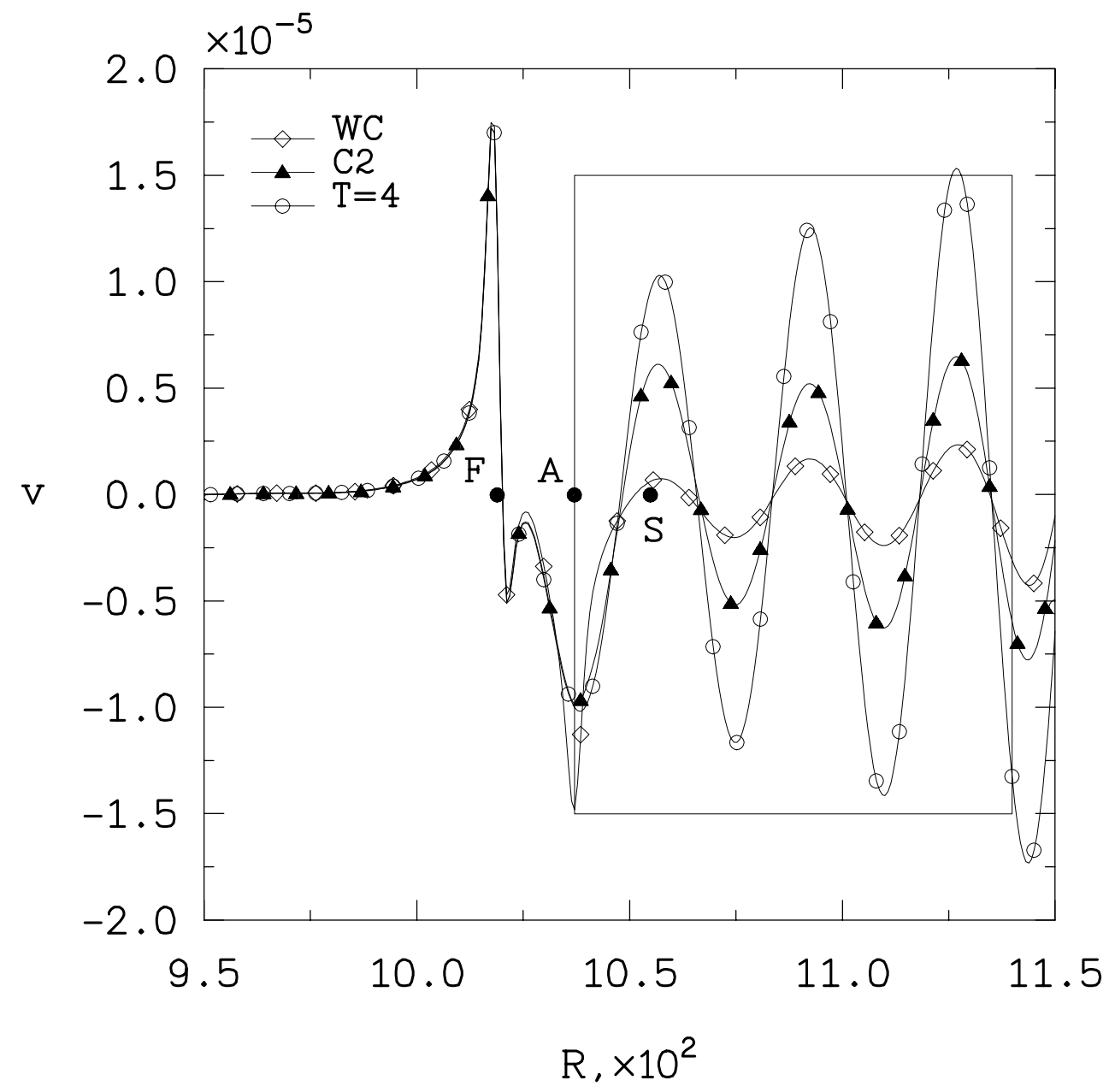

Fig. 4. Disturbance velocity with downstream distance for no control $(\mathrm{T}=4) \Gamma$ control (C2) $\Gamma$ and wave cancellation (WC) in flat-plate boundary-layer flow. (Velocity signal at $y=1.18 \delta_{o}^{*}$ from wall; $T_{1}-T_{0}=3 T_{p}$.) 

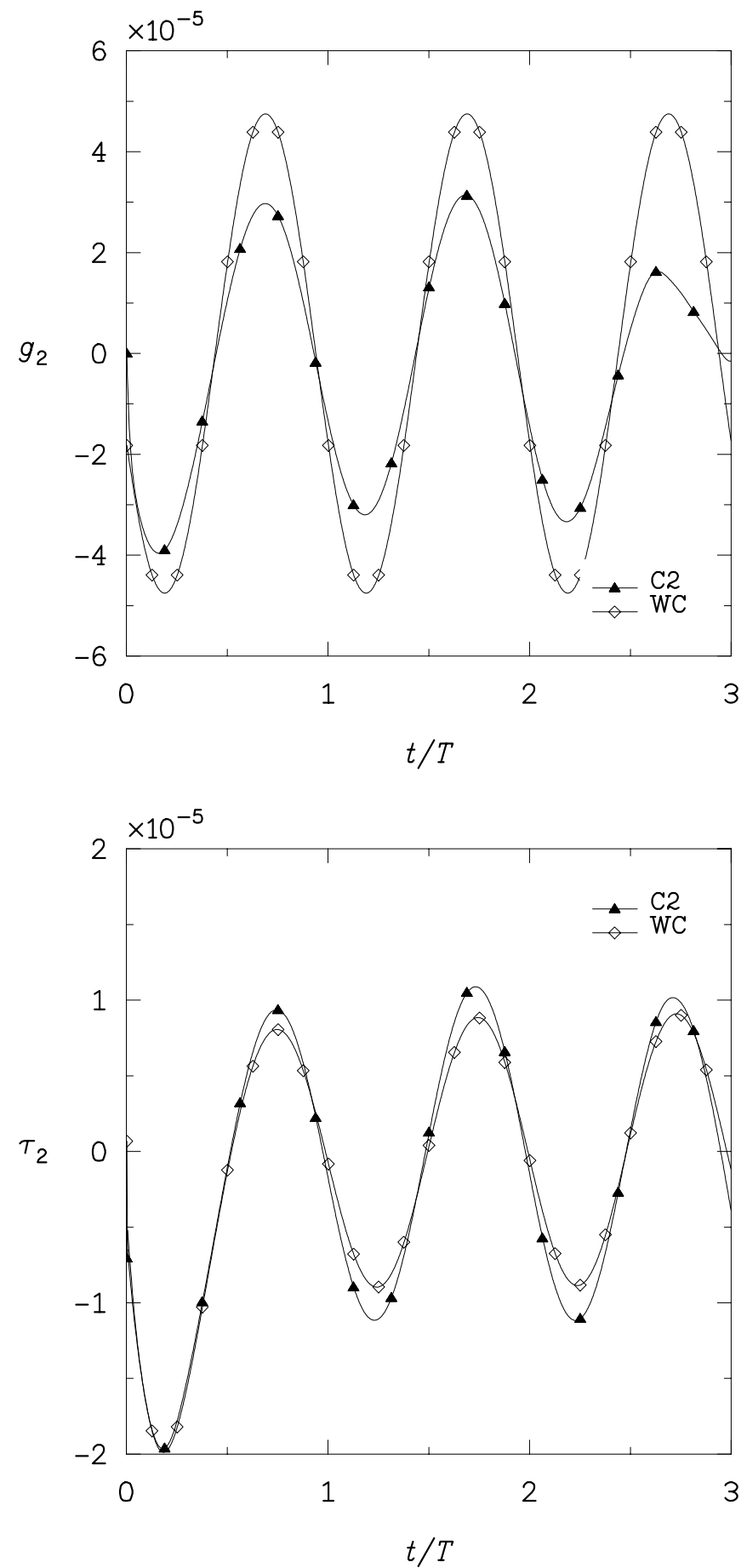

Fig. 5. Actuator response and sensor-measured shear stress for control (C2) and wave cancellation (WC) with discrete time. 


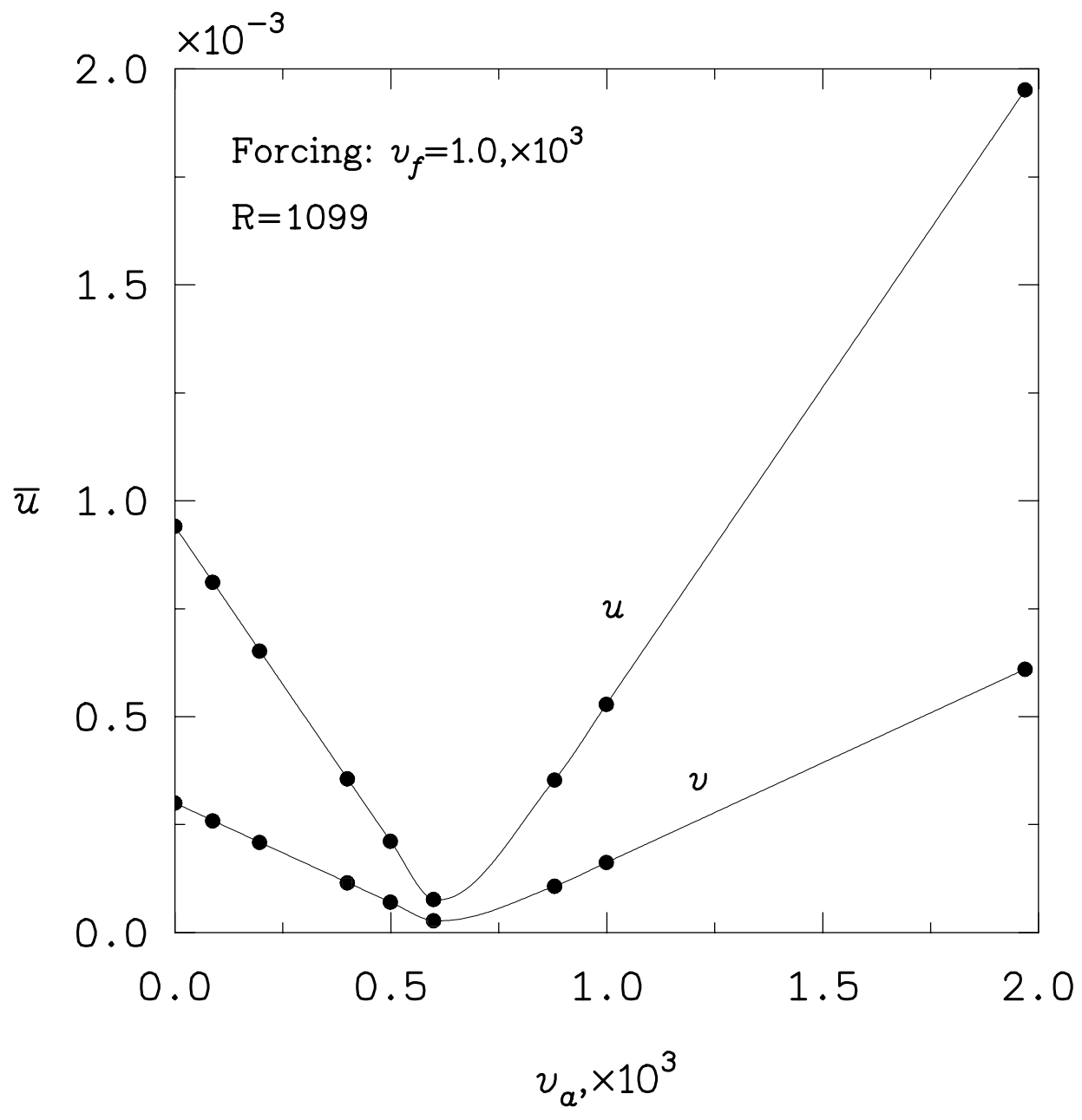

Fig. 6. Disturbance velocity resulting from variations in actuator amplitude from simulations in Joslin et al. ${ }^{21}$ 


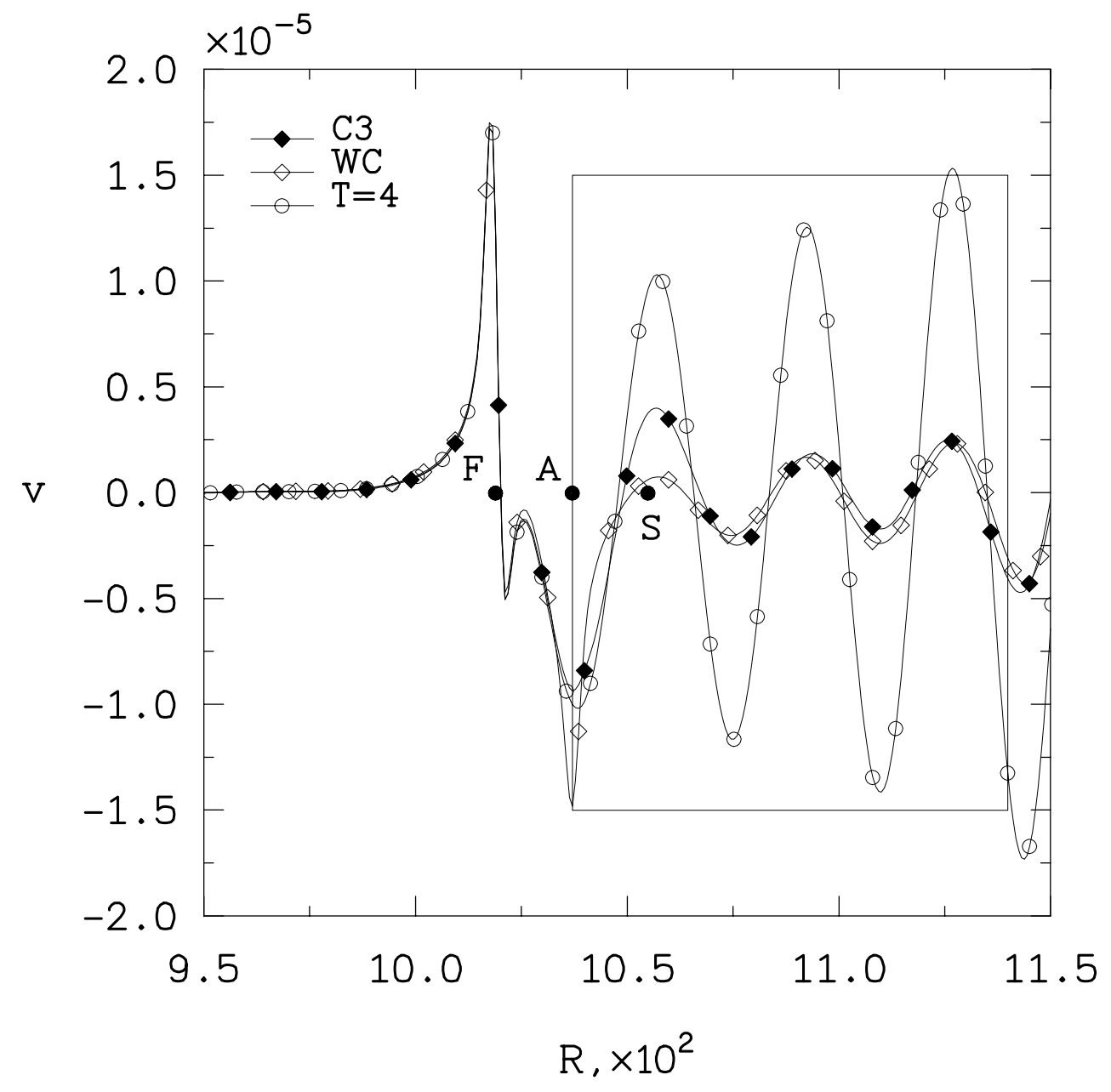

Fig. 7. Disturbance velocity with downstream distance for no control $(\mathrm{T}=4) \Gamma$ control (C3) $\Gamma$ and wave cancellation (WC) in flat-plate boundary-layer flow. (Velocity signal at $y=1.18 \delta_{o}^{*}$ from wall; $T_{1}-T_{0}=3 T_{p}$.) 

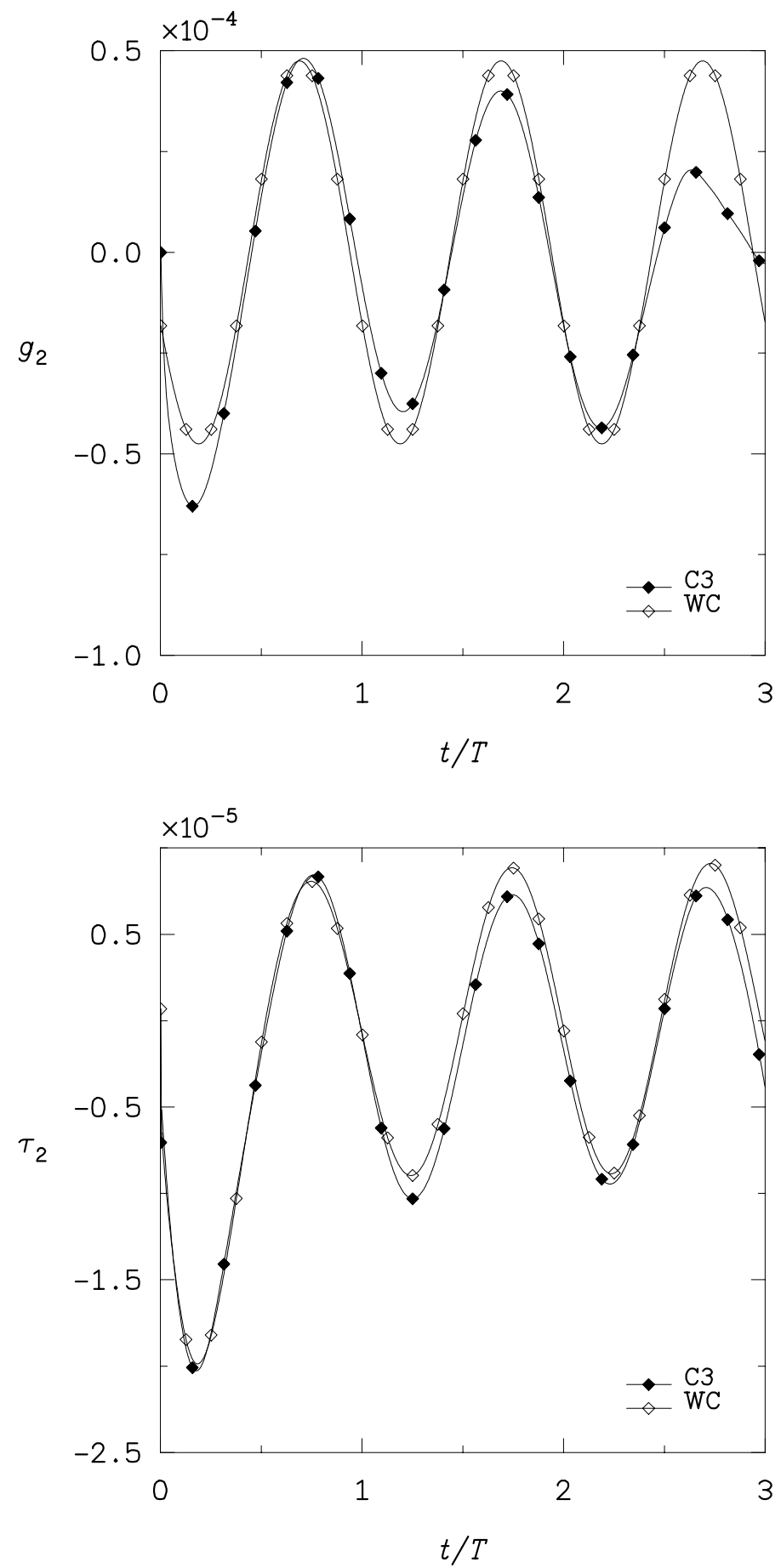

Fig. 8. Actuator response and sensor-measured shear stress for control (C3) and wave cancellation (WC) with discrete time. 